LA WAENCE LIWEAMCAE WATIONAL LABOAATOAY

\title{
Warm Dense Matter: An Overview
}

\section{Edited by \\ R. W. Lee ${ }^{1}$, D. Kalantar ${ }^{1}$, and J. Molitoris ${ }^{1}$}

\author{
With contributions from
}

K. Budil ${ }^{1}$, R. W. Falcone ${ }^{2}$, O, L. Landen ${ }^{1}$, D. H. H. Hoffmann, K. Widmann ${ }^{1}$, J. Albritton ${ }^{1}$, G. Galli ${ }^{1}$, M. Surh ${ }^{1}$, F. Rogers ${ }^{1}$, W. M. Howard $^{1}$, L. E. Fried ${ }^{1}$, C. Mailhiot ${ }^{1}$

${ }^{1}$ Lawrence Livermore National Laboratory, PO Box 808 Livermore CA 94551

${ }^{2}$ Department of Physics, University of California, Berkeley

${ }^{3}$ Gesellschaft für Schwerionenforschung, Darmstadt, Germany

\section{April 292004}


This document was prepared as an account of work sponsored by an agency of the United States Government. Neither the United States Government nor the University of California nor any of their employees, makes any warranty, express or implied, or assumes any legal liability or responsibility for the accuracy, completeness, or usefulness of any information, apparatus, product, or process disclosed, or represents that its use would not infringe privately owned rights. Reference herein to any specific commercial product, process, or service by trade name, trademark, manufacturer, or otherwise, does not necessarily constitute or imply its endorsement, recommendation, or favoring by the United States Government or the University of California. The views and opinions of authors expressed herein do not necessarily state or reflect those of the United States Government or the University of California, and shall not be used for advertising or product endorsement purposes.

This work was performed under the auspices of the U.S. Department of Energy by University of California, Lawrence Livermore National Laboratory under Contract W-7405-Eng-48. 


\section{Abstract}

This document provides a summary of the the LLNL Workshop on Extreme States of Materials: Warm Dense Matter to NIF which was held on 20, 21, and 22 February 2002 at the Wente Conference Center in Livermore, CA. The warm dense matter regime, the transitional phase space region between cold material and hot plasma, is presently poorly understood. The drive to understand the nature of matter in this regime is sparking scientific activity worldwide. In addition to pure scientific interest, finite temperature dense matter occurs in the regimes of interest to the SSMP (Stockpile Stewardship Materials Program). So that obtaining a better understanding of WDM is important to performing effective experiments at, e.g., NIF, a primary mission of LLNL.

At this workshop we examined current experimental and theoretical work performed at, and in conjunction with, LLNL to focus future activities and define our role in this rapidly emerging research area. On the experimental front LLNL plays a leading role in three of the five relevant areas and has the opportunity to become a major player in the other two. Discussion at the workshop indicated that the path forward for the experimental efforts at LLNL were two fold: First, we are doing reasonable baseline work at SPLs, HE, and High Energy Lasers with more effort encouraged. Second, we need to plan effectively for the next evolution in large scale facilities, both laser (NIF) and Light / Beam sources (LCLS/TESLA and GSI)

Theoretically, LLNL has major research advantages in areas as diverse as the thermochemical approach to warm dense matter equations of state to first principles molecular dynamics simulations. However, it was clear that there is much work to be done theoretically to understand warm dense matter. Further, there is a need for a close collaboration between the generation of verifiable experimental data that can provide benchmarks of both the experimental techniques and the theoretical capabilities.

The conclusion of this meeting is that LLNL is presently well poised to play a leading role in understanding warm dense matter as the foundation we have built in experiment/theory is strong and due to our strong connections to next generation experimental facilities. The most important recommendation is that for the SSMP to benefit the most, LLNL needs to incorporate present research activities into a consolidated programmatic effort and move forward on the experimental fronts, especially those planned for next generation facilities. 



\section{Table of Contents}

•The LLNL Warm Dense Matter Workshop ........................ 1

- Importance of the Warm Dense Matter Regime .............. 5

• Experimental Techniques ................................................... 9

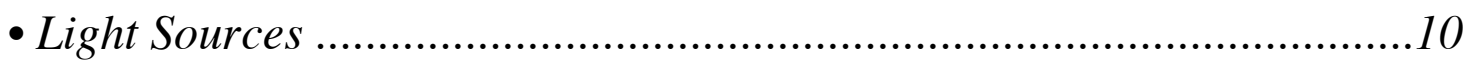

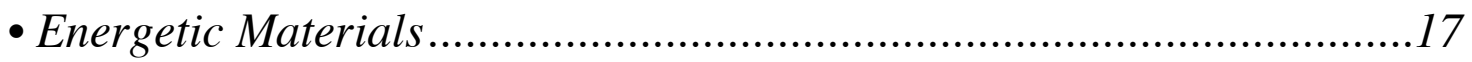

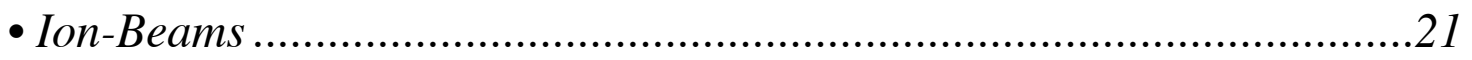

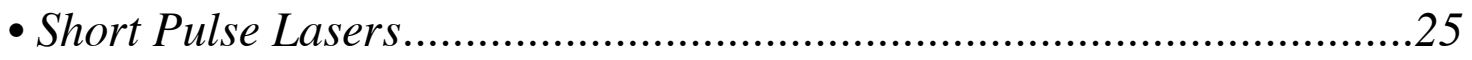

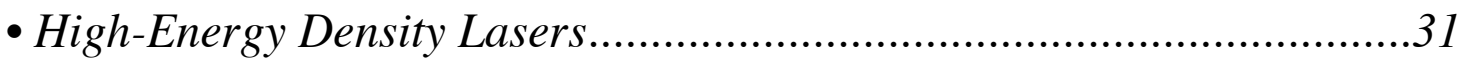

• Theoretical Review ............................................................ 37

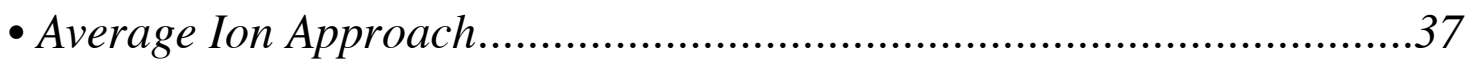

- Warm Dense Matter from First-Principles Simulations ..........................39

- Equation of State Model for Condensed Matter .....................................40

- WDM Equation of State from the Plasma Point of View ........................42

- Thermochemical Approach to Warm Dense Matter ...............................43

- Calculations of Near-neutral Population Kinetics ..................................46

- Summary of the Workshop................................................ 47

- Comments and Recommendations ...............................50 



\section{-The LLNL Warm Dense Matter Workshop}

\section{- Workshop Overview}

This workshop was organized to provide a forum for discussion and to bring together a number of distinct LLNL efforts performing research in Warm Dense Matter, the transition regime from cold materials to hot dense plasma. This meeting was able to assemble a large working group of experimentalists and theorists for two and a half days and stimulate intense discussion among them. When you can pull scientists away from their day-to-day activities at LLNL, this sort of magic can happen. LLNL clearly has the capability to dominate this emerging scientific field, but something is needed to tie the many research efforts together and have them communicate. This workshop was a first step in accomplishing that goal.

The LLNL/UC Materials Research Institute (MRI) played a central role in bringing this meeting together. Understanding the Warm Dense Matter (WDM) regime is important to the study of Extreme States of Materials, one of the MRI's primary scientific focus areas. WDM is also important to SSMP, the Stockpile Stewardship Materials Program and to the mission of LLNL. This workshop attempted to focus LLNLs efforts by examining present and future research using advanced light sources, energetic materials, ion-beams, lasers, diamond anvil cells and impact techniques. Some of these techniques are not well suited for research in WDM, but others are clear winners. Work presently being performed using energetic materials, lasers, and light sources covers a large portion of the WDM Regime. Future work using next generation light sources and upgraded heavy-ion accelerator facilities will be able to address the entire WDM region of phase space and provide a solid region of overlap with the National Ignition Facility (NIF). NIF will drive materials through the WDM regime and many targets will spend a relatively long time there, but NIF is designed to work at the cutting edge of matter at extreme conditions, not necessarily in the WDM regime. Understanding WDM is important to performing effective experiments at NIF and the goals proposed for NIF; but, WDM is not the reason NIF was built. This meeting also explored coupling different experimental techniques together. For example, creating WDM with short pulse lasers and performing EOS measurements with a light source, or using energetic materials to create WDM, then probing it using laser-produced backlighters, or with heavy-ion beams. The possibility also exists of using one technique to drive matter part way through the WDM phase space, then another to drive it to higher densities or temperatures. This workshop provided a solid review of current experimental and theoretical methods used at LLNL covering the relevant pressure, temperature, and density phase space as a function of time.

In addition to the MRI, the Institute for Laser Science and Applications (ILSA) and the Energetic Materials Center (EMC) played strong roles in this meeting. Attendees cut across the entire laboratory, with most participants coming from the PAT, CMS, DNT and NIF directorates. Over eighty people attended each day for the first two days of the 
meeting. The final day of the workshop evolved into a closed working session to assess what was accomplished and outline this report. It should be noted that a limited number of non-LLNL scientists attended the meeting from UC Berkeley, MIT, the GSI Laboratory (Darmstadt, Germany) and other institutions.

On behalf of the organizing committee and all participants, we would like to thank the Wente Conference Center for providing a splendid venue and LLNL Business Services for helping make the meeting a reality. Special recognition goes to the administrative support staff (Sharon Crowder, Joanna Allen, and Lynda Allen) which handled all the details and kept the meeting running smoothly.

Finally, we would like to acknowledge Bill Bookless and Christian Mailhiot for promoting the study of the Warm Dense Matter Regime at LLNL and recognizing both its scientific and programmatic importance.

John Molitoris and Dick Lee (Editors)

- Workshop Chairmen:

John Molitoris (EMC/CMS)

Dick Lee (PAT)

Mike McElfresh (MRI/CMS)

- Organizing Committee:

Robert Cauble (PAT)

Hector Baldis (ILSA)

Jerry Forbes (EMC/CMS)

Dick Fortner (NIF)

Dan Kalantar (ICF/NIF)

Wayne King (CMS)

Christian Mailhiot (CMS)

Carl Melius (CMS)

Craig Wuest (NIF) 


\section{-Workshop Announcement}

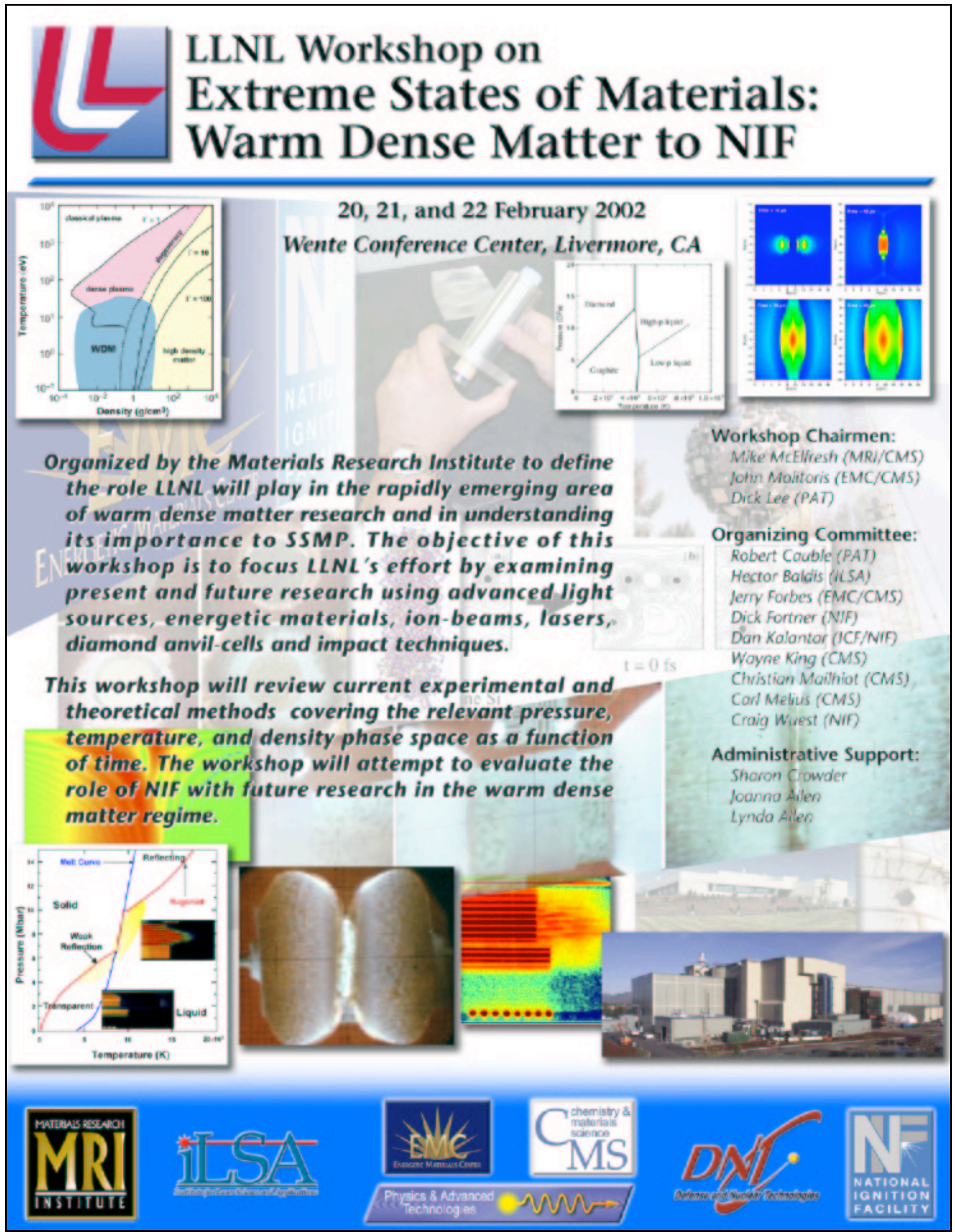




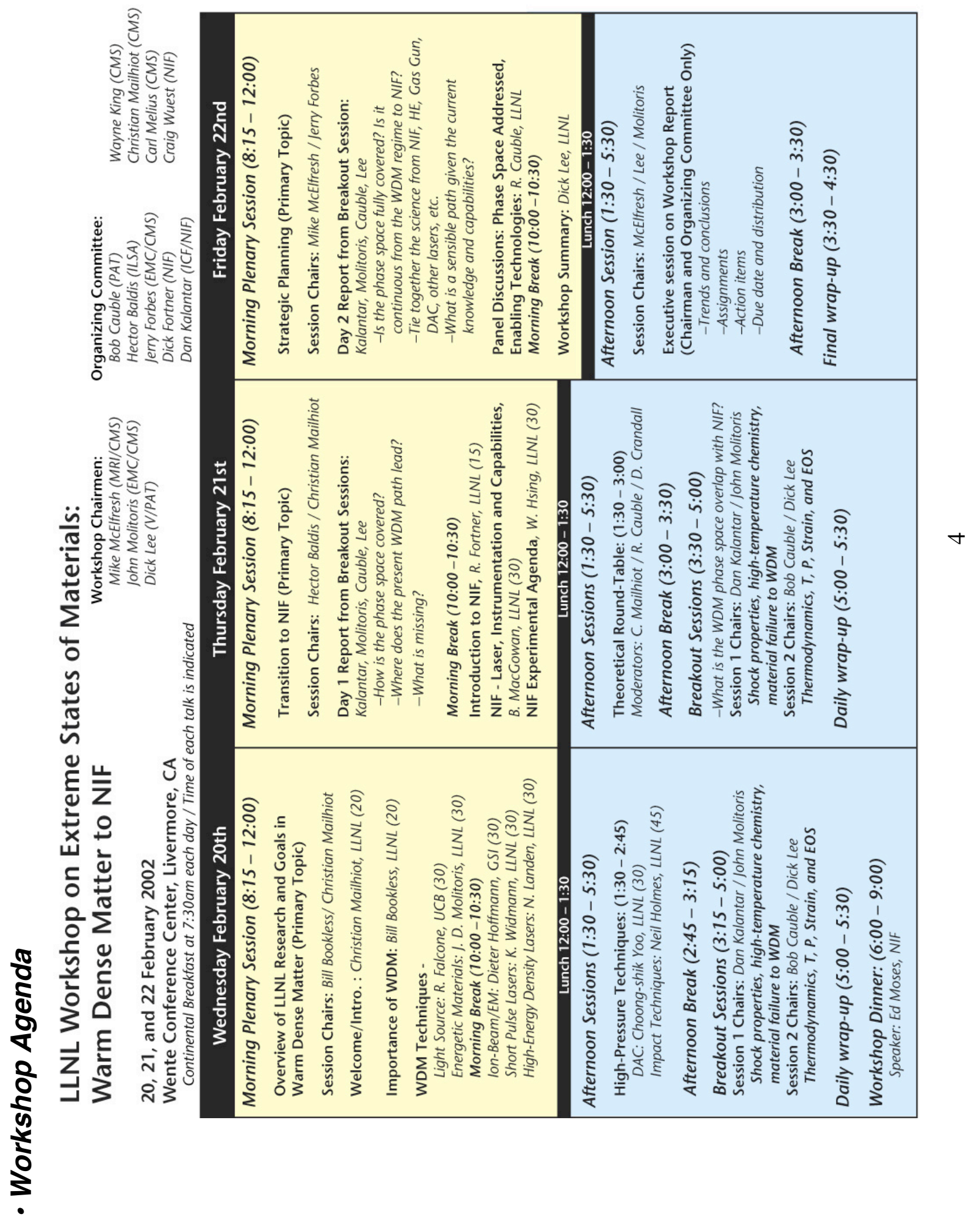




\section{- Importance of the Warm Dense Matter Regime}

\section{- Introduction}

Our confidence in ensuring the performance, safety and reliability of the stockpile is underpinned by our ability to predict, on the basis of experimentally validated sciencebased models, the dynamic response of materials under a wide range of pressure, temperature, strain, and strain rates. Without the ability to conduct nuclear testing, there exists a premium on the development of a fundamental understanding of the dynamic properties of materials - not only as they affect the performance of a baseline design but, more importantly, as they define margins and quantify uncertainties over time as a result of materials aging and/or re-manufacturing. The development of a comprehensive set of experimentally validated predictive capabilities to assess the effects of materials properties on stockpile performance margins and uncertainties is at the heart of the stockpile stewardship program and forms the basis of a scientific strategy to mitigate these uncertainties.

The dynamic response of materials can be broadly described in terms of two classes of properties: thermodynamic properties and mechanical constitutive properties. Thermodynamic properties are determined at the quantum and atomic scales. They include equation-of-state (EOS), melt, phase transitions, phase diagrams, etc. On the other hand, mechanical constitutive properties are governed by phenomena occurring across various length scales - from the quantum-level scale to the continuum - and are dominated by the collective behavior of defects and the evolution of the materials microstructure. Mechanical constitutive properties include strength and plasticity, fracture and failure, spall, ejecta, etc.

The thermodynamic properties of materials are independent of the details of their microstructure and their determination does not require a scientific approach based on a "multi-lengthscale" strategy bridging the quantum and continuum scales. The experimentally validated prediction of the thermodynamic properties of materials under extreme conditions of pressure and temperature has formed the basis of a longstanding, vigorous program at Lawrence Livermore National Laboratory (LLNL). Scientifically diverse theoretical approaches and experimental facilities have been deployed to predict and characterize, respectively, states of matter in regimes of high-pressure and hightemperature. The first figure below provides a general indication of programmatically relevant density-temperature regimes $(\rho-T)$ accessed by various experimental facilities.

Modern theoretical frameworks have been extremely successful in predicting the properties of condensed matter systems at low temperature (typically below the melt temperature, $T_{m}$ ) and plasmas at high temperature (typically well above the Fermi temperature, $T_{f}$ ). However, there exists an important regime within the $\rho-T$ phase space where "warm" states of matter are inadequately described by either conventional condensed matter physics-based or plasma physics-based theories, and where the greatest 
uncertainties exist in our ability to predict the thermodynamic properties of matter. From a condensed matter physics perspective, warm dense matter refers to states of matter with solid-like densities and temperatures comparable to $T_{F}$. From a plasma physics perspective, warm dense matter refers to states of matter that are plasma-like, but that are too dense and/or too cold to be adequately treated by standard plasma physics approaches. Consequently, this warm dense matter regime - roughly spanning the energy range between $T_{m}$ and $T_{F}$ - defines states of matter between solids and plasmas. The second figure below indicates the region of $\rho-T$ phase space defining states of warm dense matter. Note that this definition works, roughly speaking for the condensed matter phase of the regime; but, it is clear that a strongly coupled plasma with a $\Gamma$ of, say, $\geq 2$ will be a very difficult phase to study and is in the WDM regime from the plasma point of view. Thus, the $T_{F}$ part of the definition does not work for the plasma end of the regime and we need to surplant the concept of $T_{f}$ with the strong coupling parameter, $\Gamma$, which represents the ratio of the interaction energy to the kinetics energy of the system.

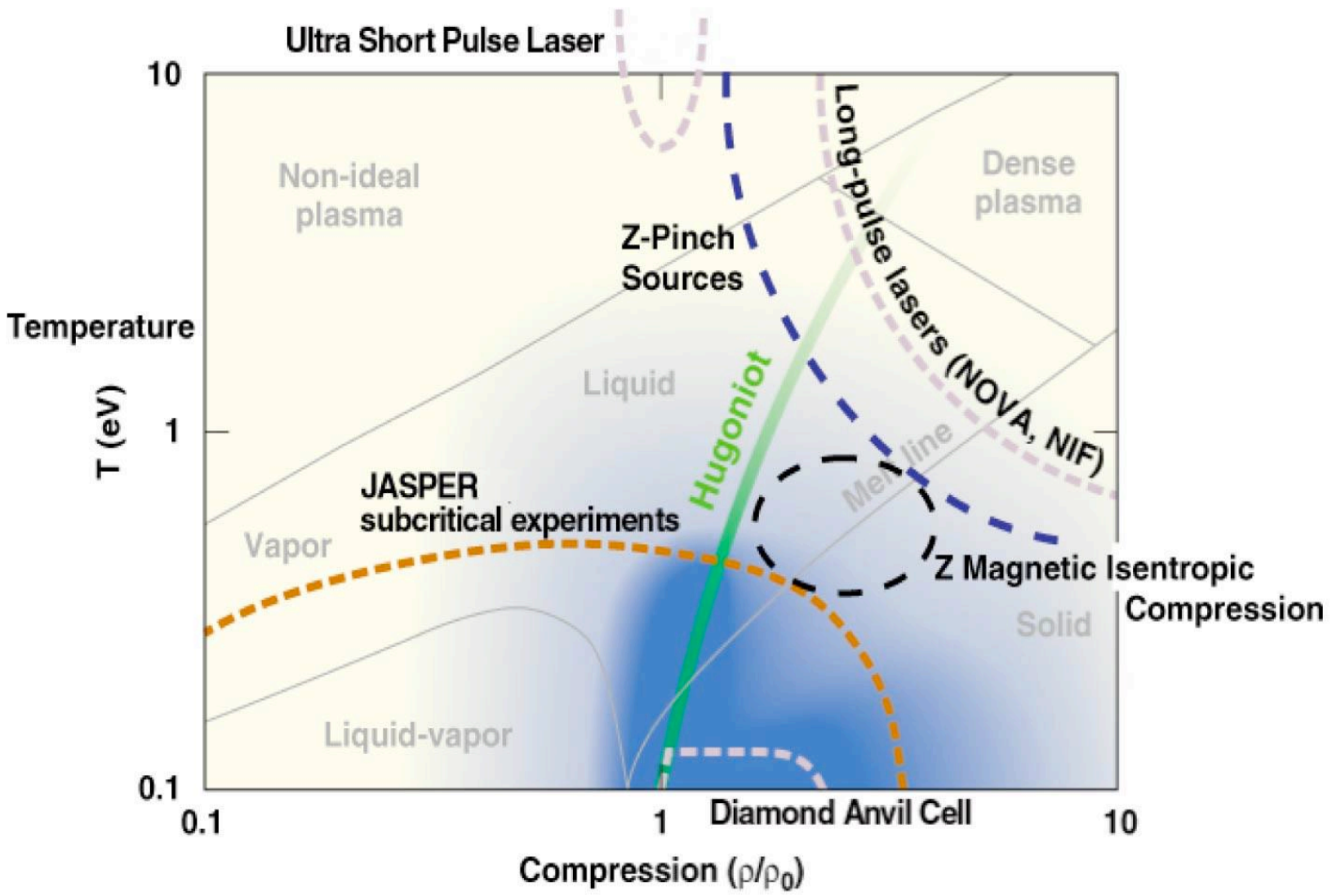

Figure: Summary indication of experimental facilities required to create states of matter spanning programmatically significant density-temperature $(\rho-T)$ regimes. JASPER refers to the $\underline{J}$ Jint $\underline{A}$ ctinides $\underline{S}$ hock $\underline{\text { Physics }}$ Experimental Research facility - a two-stage gas gun designed to perform shock physics experiments on special nuclear materials - at the Nevada Test Site. $Z$ refers to the pulsed-power $Z$-pinch accelerator at Sandia National Laboratory, NM. 
Scientifically, the warm dense matter region of $\rho-T$ phase space is attracting considerable - and rapidly increasing - interest, both theoretically and experimentally. Within the experimental condensed matter physics community, interest has been stimulated by the emergence of new techniques for generating strong shock waves in materials, and new methods for confining and interrogating "warm" samples at high pressures. Within the plasma physics community, the development of novel sources enabling experimental access to plasma-like states of matter at low temperature and high density has lead to emerging research opportunities at the forefront of the field. Theoretically and computationally, recent advances in finite-temperature ab initio electronic structure methods beyond mean-field theories, coupled with the unprecedented surge in computer power afforded by ultra-scale computing platforms, have lead to important new developments in our ability to predict the thermodynamic properties of warm states of matter.
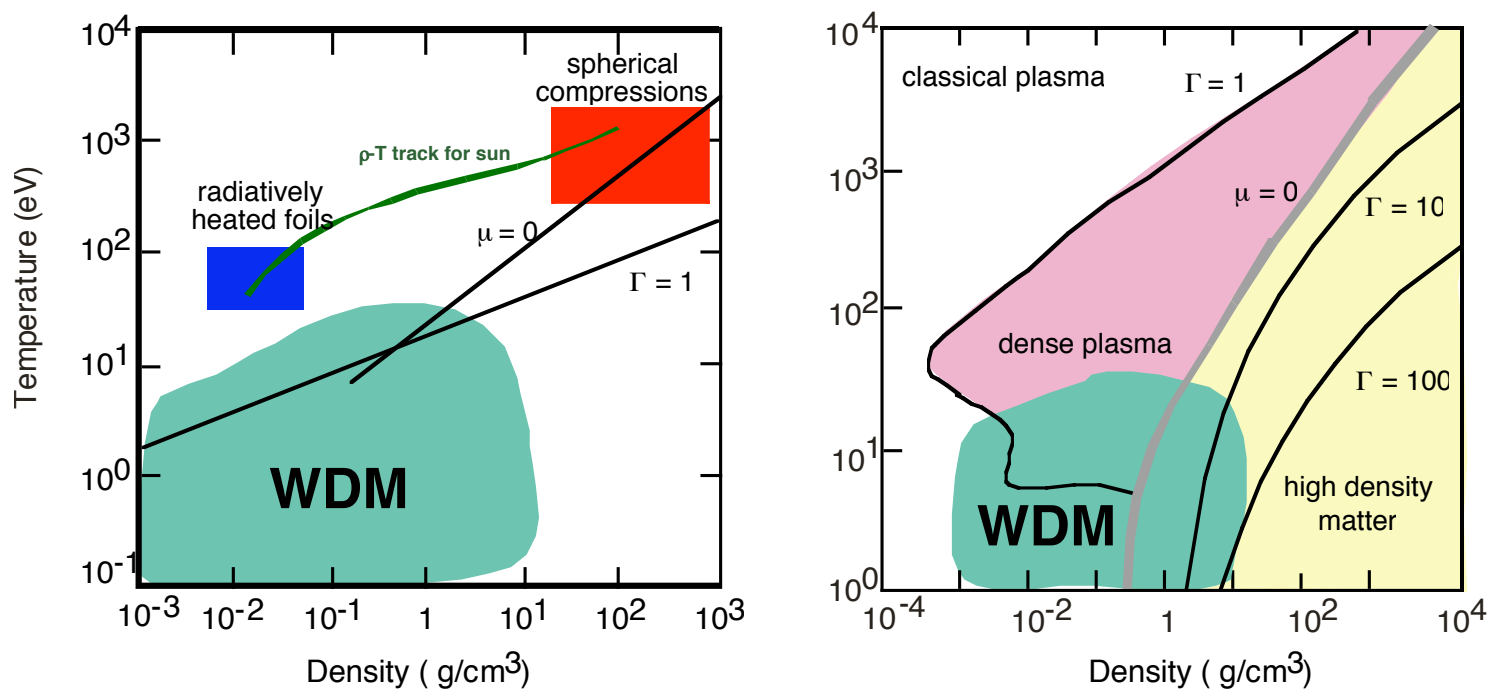

Figure: The temperature-density phase diagram is shown for hydrogen (left) and aluminum (right). The relevant regimes are noted, as are the various values of the coupling parameter $\Gamma$. The regions of greatest uncertainty are roughly noted by the green areas. Also indicated is the region where degeneracy will become important, to the right of the line where the chemical potential $\mu=0$.

\section{- Emerging Opportunities for LLNL}

The investigations of states of matter in the warm dense regime - a novel field of research - offer unprecedented opportunities, both within the laboratory and the community at-large, to explore new scientific frontiers at the intersection of various disciplines: condensed matter physics and the science of materials under extreme conditions, high-pressure physics and chemistry, shock physics, strongly-coupled plasma physics, planetary science, astrophysics, inertial confinement fusion, and others.

More specifically, the study of warm dense states of matter is of scientific and programmatic significance for LLNL: 
- LLNL's broad core scientific competencies in high-energy density science, highpressure physics and chemistry, shock physics, inertial confinement fusion, highenergy and ultra-short pulse laser science and technology, weapons research, and high-performance simulations of complex physical systems directly overlap with the technical elements necessary to make important scientific discoveries in the investigations of states of matter in the warm dense regime. LLNL holds a natural leadership position in this emerging field.

- The study of warm dense states of matter is directly aligned with LLNL's programmatic mission needs. In fact, the $\rho-T$ regimes of largest errors and uncertainties in our treatment of matter under extreme conditions of pressure and temperature overlap the warm dense regime.

While LLNL currently supports efforts in the field of warm dense matter science, an overarching, coordinated, and integrated program would enhance the benefits to the laboratory's programmatic mission needs and accelerate scientific discoveries in this emerging field. In order to "take stock" of current laboratory efforts in the field of warm dense matter science and identify key scientific challenges, a workshop was organized to bring together the laboratory community active in this field.

\section{- Objectives of Warm Dense Matter Workshop}

The objective of the Warm Dense Matter workshop was to bring together the LLNL technical community active in the field in order to identify the key scientific challenges - theoretical, computational, and experimental - underpinning the investigation of matter in the warm dense regime. Another important aspect of the workshop was a comprehensive and comparative discussion of the various experimental facilities and theoretical capabilities to be brought to bear in these investigations.

In particular, the following topics were discussed:

- Experimental capabilities and facilities enabling the synthesis and confinement of warm dense states of matter and advanced diagnostics required for the characterization and interrogation of such states. These experimental capabilities include radiation-synchrotron sources, energetic materials, ion beams, shortpulse lasers, high-energy-density lasers, static high-pressure diamond-anvil cells, and mechanical impact techniques such as utilized in gas-gun launchers.

- Theoretical approaches and computational capabilities enabling the prediction of the thermodynamic properties of matter in the warm dense regime. These approaches include quantum-based finite-temperature methods based on densityfunctional theory, rate equation theory, kinetic theories, finite-temperature average-atom method, and various plasma physics-based theoretical approaches.

This document summarizes these discussions and serves as the basis to establish and implement a forefront coordinated and integrated laboratory-based program in the field of warm dense matter science. 


\section{- Experimental Techniques}

\section{(J. D. Molitoris)}

Presentations were given on five proven experimental techniques that clearly provide access to the warm dense matter regime. Using the temperature - density diagram for aluminum as an example, the phase space covered by each of the techniques overlaps to cover the WDM regime as illustrated in the figure below. It was clear that the techniques were synergistic and could be combined on common experiments, for example, to use one technique to generate WDM and another to probe it. Light sources presently provide a good way to probe laser-produced WDM, while heavy-ions and lasers can diagnose the properties of WDM made with energetic materials. The GSI heavy-ion facility even has a high-explosive firing tank installed on one of its beam lines with this purpose in mind and new short pulse lasers capabilities are planned at several light sources.

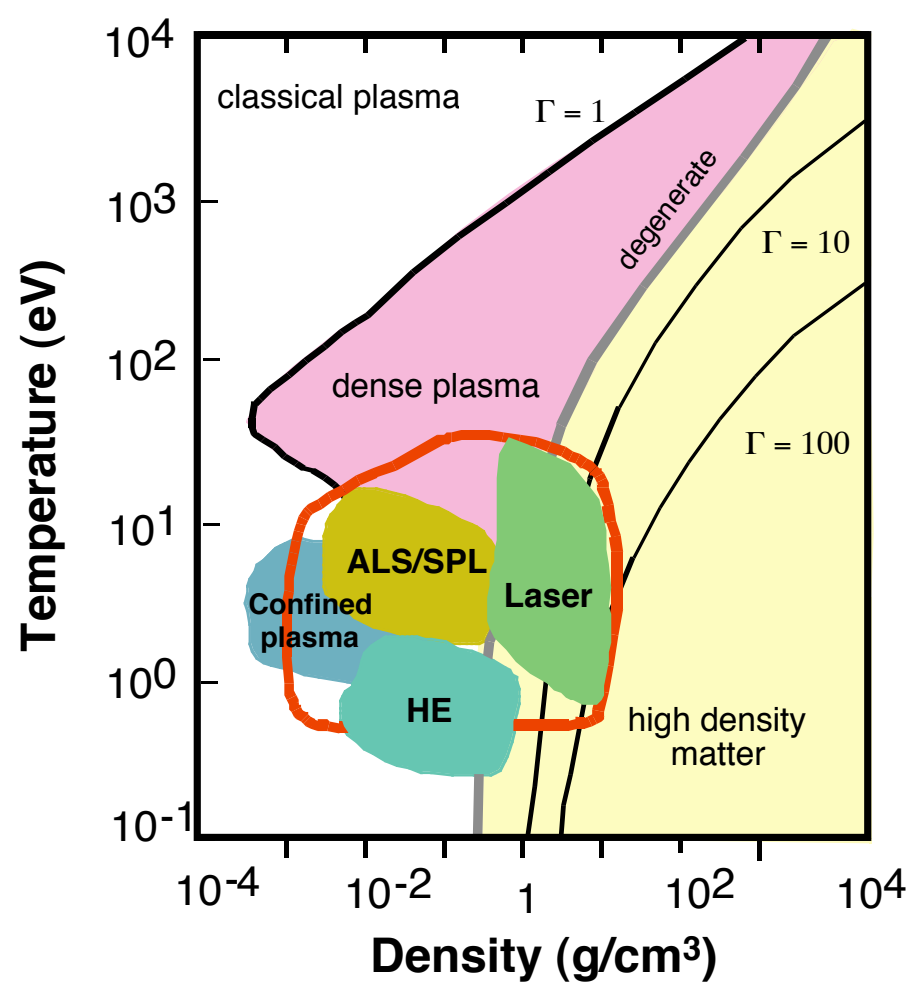

Figure. Using the Al $\rho$ - T diagram as an example, the plot shows the phase spaced accessed by experimental techniques presently being used to investigate WDM.

Each of the techniques had its unique set of advantages and disadvantages. Heavy-ion facilities can best address the WDM properties of heavy metals, but even future incarnations, will not be able to address temperatures below about $17 \mathrm{eV}$. The energetic materials technique developed at HEAF is probably best utilized for the study of WDM equilibrium conditions due to the long time duration and amount of warm matter 
produced. However, this technique is presently limited to temperatures less than a few electron volts. It is interesting to note that all of the experimental work in progress has phase space overlap with the phase space accessible to NIF.

The future of WDM research is in NIF, $4^{\text {th }}$ Generation Light Sources, and the GSI HeavyIon Accelerator Upgrade. Short pulse lasers and energetic material techniques will play a role in WDM, but the large facilities are where the action will be focused. They will have infrastructure and diagnostic capabilities to pursue complex WDM experiments. However, these facilities are not yet online and there is much work to be done in the meantime.

The fact that LLNL has an active role in all five WDM experimental techniques is astounding. This puts us in a unique experimental position to be a major player in this field, assuming we take the ball and run with it. There was a general consensus at the workshop that the experimental components saw the value in working together, but as NIF is only one of the future focal point, LLNL needs to strongly couple with the other two (light sources and heavy-ions). That coupling should start now if LLNL is to be a major player in WDM, but consistent programmatic funding is necessary to tread this path. Discussions and highpoints of the experimental techniques follow.

\section{- Light Sources}

\section{(R. Falcone)}

The use of x-ray light sources for the study of warm dense matter has at its base the appeal of an intense spectrally tunable source of x-rays. This source can be used to probe a warmed sample to provide information on the structure of the matter, the charge states of the matter, and/or its opacity. In all these cases, x-ray light sources can be seen as an efficient method to obtain information on WDM. Here we outline the capabilities of $\mathrm{x}$ ray light sources that have made impacts in areas related to WDM research and those that will, we believe, allow substantive advances in the future. In this regard we point out that by its very nature, warm matter created by short pulses is transient, requiring diagnostic resolution times on the order of a picosecond, which is the limitation of most current $\mathrm{x}$ ray light sources and at the edge of the useful regime. Furthermore, the fact that we have finite temperature indicates that there will be signal coming from the samples adding an additional noise source to the usual ones associated with x-ray light source experiments. Thus, we will emphasize both short time and intense $x$-ray sources here and discuss below what is currently referred to as ultrafast $x$-ray sources. The phase space covered by this experimental technique is near solid density and up to temperatures of $1 \mathrm{eV}$. The WDM is usually created by using short pulse lasers to initiate phonon modes. 


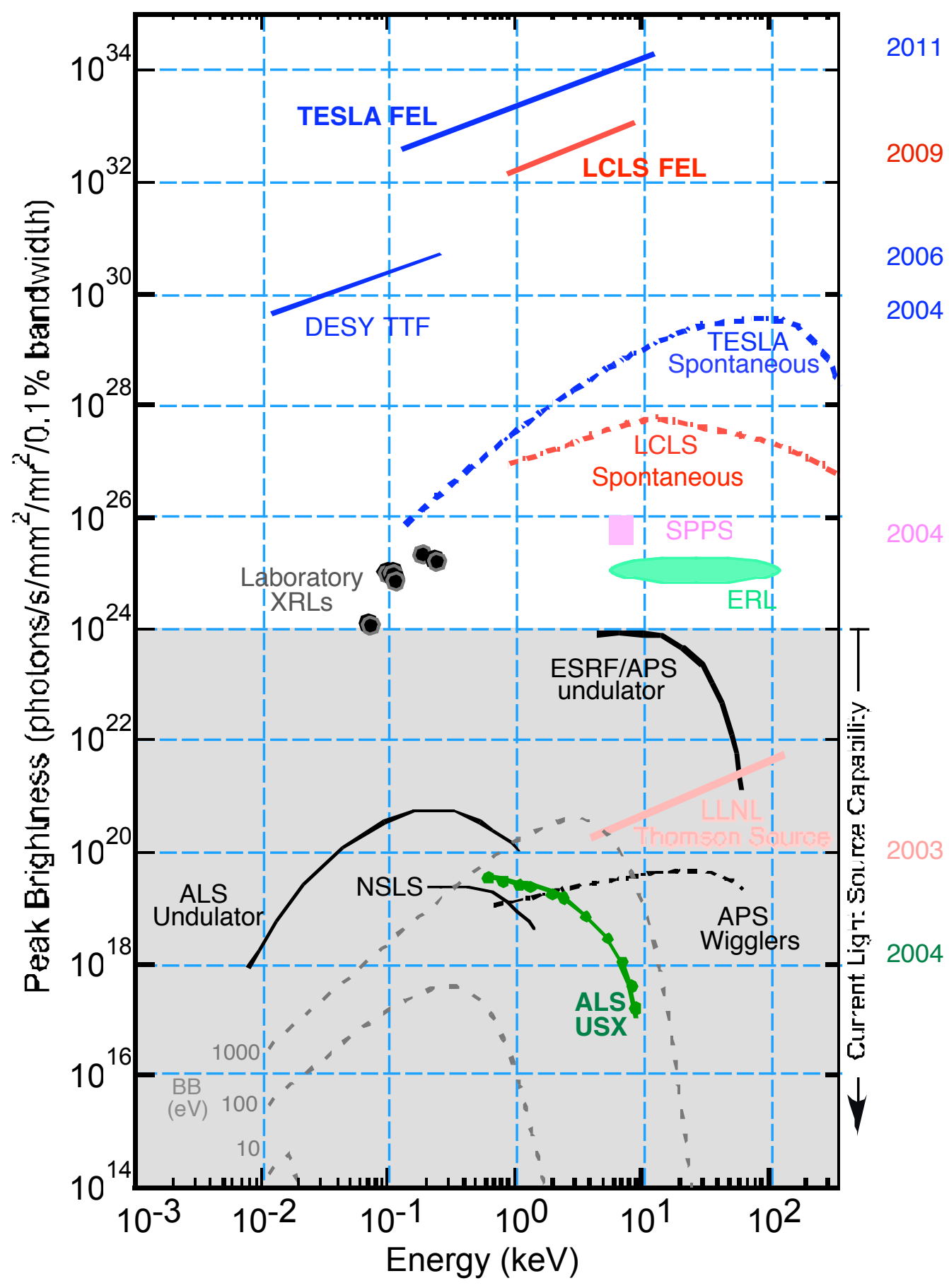

Figure: A comparison of the x-ray sources discussed in the text in units employed in synchrotron light source facilities. The high repetition rate of the current sources are intended to provide photons on average while the figure of importance here is peak brightness emphasizing the individual photons per bunch needed for warm dense matter studies. 
To attempt to make the discussion of the x-ray sources understandable, we show in the figure above the range of potential sources. The first thing to note is that although drawn on a plot of peak brightness versus photon energy in $\mathrm{keV}$, there is a clear timeline for various future sources. We have represented the existing sources as various black lines and indicated the limits of current $3^{\text {rd }}$ generation synchrotron light source technology with the gray area at the lower part of the plot. The use of peak brightness, a light source unit, defined as the number of photons per second per $\mathrm{mm}^{2}$ per milliradian ${ }^{2}$ $\left(\mathrm{mr}^{2}\right)$ per $0.1 \%$ bandwidth (BW) allows one to compare a large variety of x-ray sources. The various acronyms for current sources are as follows: ALS (Advance Light Source at LBNL); ESRF (European Synchrotron Radiation Facility at Grenoble, France); APS (Advanced Photon Source at Argonne); NSLS (National Synchrotron Light Source at Brookhaven). Indicated in figure 1 below are the proposed $4^{\text {th }}$ generation synchrotron based sources in various colors with the same color indicating the possible start date. The various acronyms for proposed sources are as follows: USX (UltraShort X-ray source at LBNL); SPPS (Sub-picosecond Pulse Photon Facility at SLAC); ERL (Energy recovery LINAC several proposed); TTF (Tesla Test Facility at DESY in Hamburg, Germany); LCLS (LINAC Coherent Light Source at SLAC), and TESLA (TeV Energy Superconducting Linear Accelerator at DESY near Hamburg, Germany).

Finally, there are three additional sets of data on the plot to provide contact with more standard laser-based and DOE/DP x-ray sources. Thus, we have added the peak brightness of the various blackbody radiators for 1000,100 , ands $10 \mathrm{eV}$ as dashed gray lines (e.g., $1000 \mathrm{eV} \mathrm{BB}$ ). One can then understand the utility of the light sources as probes and grasp the difficulty one would have trying to reproduce these $\mathrm{x}$-ray sources, at least as probes. Next we include several actual and potential laboratory x-ray lasers (XRLs) as black circles. Finally, we include the potential high brightness Thomson Source currently being developed at LLNL. Note that not all of these X-ray sources will be discussed in detail below, but they are noted here for completeness.

\section{- $3^{\text {rd }}$ Generation Synchrotron Based Light Sources}

Light sources have provided us with some of the first experimental results in the WDM regime. The $3^{\text {rd }}$ generation light source based experiments that contact the warm dense matter regime are

-Warming a crystal to temperatures below the melt to induce phonon modes measured in diffraction. This is the schematic experiment used below.

-Warming a crystal to temperatures above the melt to measure the loss of order

-Warming matter to above the melt to which the solid-liquid phase transition 
-Warming matter to investigate the processes involved in surface ablation.

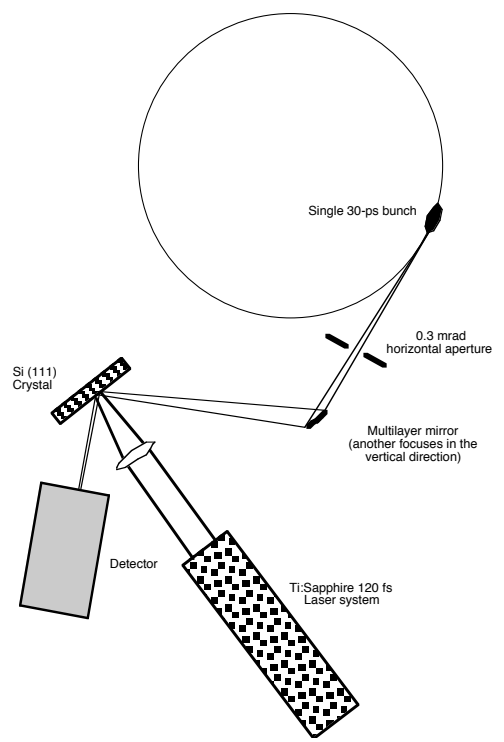

Figure: X-ray diffraction set-up using the ALS as the probe beam of a laser heated crystal. Time dependent diffraction is recorded on a streak camera. Note that this set-up can be considered an experimental schematic of all the proposed experiments we discuss. That is, a Light Source is the probe of a sample that is perturbed at various strengths with a short pulse laser and the time-dependent response is monitored by a fast $x$-ray detector.

A schematic diagram of a generic experiment is shown in the figure above. Here an $80 \mathrm{ps}$ pulse of 3-keV x-rays from a single bunch of the ALS is focused with approximately unit magnification onto, and diffracted from, a silicon (111) crystal. During the x-ray diffraction the crystal is irradiated with a 120 -fs laser pulse of $800-\mathrm{nm}$ light, resulting in volumetric heating of the crystal. Upon melting, the crystal will no longer diffract efficiently. The $\mathrm{x}$-rays are detected in two modes: a) using a time-integrating (over the single 30-ps bunch) single photon counter (CCD), and, b) using a time resolving x-ray streak camera, with an intrinsic resolution of 2-ps, and an ultimate resolution of 200-fs in single-photon counting mode. The time scale for crystal melting will be detected in the first case in a sequence of shots, altering the delay between the laser beam and the synchrotron beam for each laser shot. For the second case, a large number of shots can be taken at the same laser/synchrotron delay. These experiments have also been performed at the APS and ESRF, see figure above for the specific peak brightness of these synchrotrons.

Following are typical experimental characteristics taken from the experiment performed at the ALS. The x-rays are produced from a single 30-ps bunch. At $1.9 \mathrm{GeV}$ their divergence is of order $0.3 \mathrm{mrad}$ - instantaneously this holds in both the horizontal and vertical directions. In order to ensure that a pulse of order $30 \mathrm{ps}$ is incident on the crystal, the entrance aperture in the horizontal direction is set to $\sim 0.3 \mathrm{mrad}$, i.e., the natural divergence of the beam. If we collect a larger horizontal divergence, we will start to increase the effective pulse length, unless we slant the target considerably. This effect is 
due to the fact that the bunch moves through $2 \pi$ in $656 \mathrm{~ns}$, and therefore sweeps out 0.3 mrad in 30 ps. Slanting the target to utilize more horizontal divergence is not practical. The final laser spot sizes are of order 100- $\mu \mathrm{m}$ (to match the streak camera slit), whereas a 30 -ps pulse is $\sim 1 \mathrm{~cm}$ long.

The area of crystal to be irradiated is dictated by two factors. Firstly, the 200-mJ, 120-fs pulse from the Ti:Sapphire laser can melt a region of the crystal of less than 1-mm diameter. Secondly, for the second mode of $\mathrm{x}$-ray detection, i.e., the streak camera, the $\mathrm{x}-$ rays need to be focused to a spot of order $100-\mu$ m diameter to maximize sensitivity, as this is the width of the entrance slit to the camera. Thus, we will need to focus the beam using a pair of crossed multilayer mirrors. The $100-\mu \mathrm{m}$ spot is of the same order as the electron beam size, therefore we are, in principle, able to utilize the full $0.3 \mathrm{mrad}$ of useful horizontal divergence. This will require a one-to-one magnification system. It can be shown that for the example experiment the number of photons from the synchrotron will be a function of the particular beamline and ranges from $\sim 2 \times 10^{3}$ to $\sim 3 \times 10^{5}$, while the number of recorded photons after diffraction from the crystal varies from $\sim 3 \times 10^{2}$ to $\sim 5 \times 10^{4}$.

We describe the short pulse laser coupled to the schematic experiment for two reasons. First, the laser is an integral part of the experimental setup and, second, it is believed that short pulse lasers will play a role in many future warm dense matter experiments on large-scale x-ray sources. The laser source is a relatively conventional $\mathrm{TiAl}_{2} \mathrm{O}_{3}$ laser system used for the production of ultrashort laser pulses $(<100 \mathrm{fs})$ at a wavelength of 0.8 $\mu \mathrm{m}$ and has a pulse energy of up to 1 Joule. Laser technical specifications were not that demanding: we require a system capable of ultrashort pulse operation, $100 \mathrm{fs}$ or less as described above, with a per pulse energy of about $1 \mathrm{~mJ}$ and a repetition rate as high as possible. This system typically involves an oscillator and regenerative amplifier.

The generic experiment discussed here requires the following instrumentation:

- Si (111) monochromator crystal to select a single wavelength with a spectral bandwidth of $1 \mathrm{~m} \AA$.

- Ti: $\mathrm{Al}_{2} \mathrm{O}_{3}$-based $150 \mathrm{fs}, 1 \mathrm{KHz}, 800 \mathrm{~nm}$ laser to warm the sample

- Circuitry to synchronize the laser to the individual electron bunches within the synchrotron ring with jitter less than 5 ps.

- Time-resolved x-ray diffraction measurements using a streak camera detector triggered by a GaAs photoconductive switch.

- CCD camera to record the x-ray streak projected onto a phosphor screen. All recorded data are averaged for long periods. For example, in the schematic experiment shown the period was 60 s corresponding to 60,000 shots. The resulting temporal resolution of the camera is $3 \mathrm{ps}$; this is monitored using an ultraviolet femtosecond pulse split off from the main pump laser. The entire time history of the diffracted signal following laser excitation is measured at once, in contrast to more typical pump-probe geometries. 


\section{- LINAC Based Sources}

Radiation from a FEL is similar to the radiation extracted from a standard optical laser in that it has high power, narrow bandwidth, and diffraction limited beam. There are major differences, of which, a major one is the gain medium: in the FEL the amplification medium is composed of free, not the bound, electrons that are stimulated. In the FEL the electrons propagate through a long periodic magnetic dipole array, i.e., an undulator, and the interaction with the electromagnetic radiation field leads to exponentiation of the free electron emission. When the initial radiation is an internal field caused by the spontaneous emission of the undulator the process is called SASE for Self Amplified Spontaneous Emission. This is the basis for x-ray FELs proposed for the future. Note that since the electrons are not bound the wavelength of the FEL is tunable.

For warm dense matter research the main advantage of the X-ray and XUV-FEL is that these have very high photons number per sub-picosecond bunch generating peak brightnesses that are roughly 10 order of magnitude higher than other light source methods, see figure. The repetitions rate will be up to $100 \mathrm{~Hz}$ and these can be viewed as large-scale user facilities, although being linear they do not have the advantage of the number of users that can be adapted to the current synchrotron ring.

There are of course difficulties associated with the development of a sources as novel as these FELs. Some of the issues are sample damage, power/energy handling, multiple user accessibility, synchronization of the FEL pulse to other systems, e.g., laser and detectors, and shot-to-shot reliability. All of these will be challenges but there is a strong case to be made for using, what is essentially an x-ray laser, for warm dense matter research.

In addition to the FELs there is another technology that is being proposed as the next generation light sources. These are based on LINACs also, but here the high repetition rates of the current synchrotrons light sources is attained, while the use of the LINAC permits the pulse length to be sub-picosecond and the recirculating nature of the device accommodates multiple users around the system. The idea behind the energy recirculating LINAC, ERL, is to make a device that solves one of the two major drawback for current light sources: the time structure of the ERL will clearly go toward the $100 \mathrm{fs}$ regime. This, then, provides an interesting advance from the point of view of warm dense matter as fast time response is necessary. On the other hand, the low number of photons per bunch will make single shot experiment difficult. In essence these light sources are the incremental improvement on the current light sources.

\section{- Fast Detector Technology}

It should be clear that the need to measure rapid changes in the creation and eventual deterioration of a warm dense matter sample require sub-picosecond time resolution. In all of the above discussions it was assumed that the method of obtaining these data would rely on sub-picosecond x-ray sources. However, it is also possible to obtain the necessary time resolution by using an $\mathrm{x}$-ray detector that has sub-picosecond response. Additionally, since we will probe with x-rays one could use cross-correlations method 
wherein a short pulse optical laser interacts with the x-rays of interest to provide a subpicosecond capability. We will not address this latter method here.

In direct x-ray measurements, a leading diagnostic candidate seems to be the development of an x-ray streak camera with sub-picosecond resolution that could be synchronized to a light source. As sub-picosecond x-ray streak cameras have been used in the past on laser generated plasma sources to measure emission line characteristics this technological advance is clearly possible. Further, x-ray streak cameras of near 1 picosecond resolution have been coupled to synchrotron to measure various phenomena. Nonetheless, the sub-picosecond x-ray streak cameras have been used primarily in the single shot mode on laser-based experiments.

The emphasis from our point of view will be on studying the dynamics of processes in finite temperature dense matter and requires the development of sub-picosecond x-ray diagnostics. As the (single shot) resolution of current x-ray streak cameras is limited to $\sim 600$ fs covering a time window of $\sim 100 \mathrm{ps,} \mathrm{improvements} \mathrm{are} \mathrm{necessary.} \mathrm{The} \mathrm{current}$ limitation arises due to the fact that the electrons liberated from the photocathode have a finite energy distribution and disperse temporally prior to being swept by a voltage ramp across the detector. This dispersion can be reduced by increasing the extraction field at the cathode, as the majority of the dispersion occurs while the electrons have low energy and are moving with low velocity. On the other hand, with $10 \mathrm{kV}$ across a gap of $250 \mu \mathrm{m}$ the transit time difference between electrons with initial energy $0 \mathrm{eV}$ and $2 \mathrm{eV}$ is $\sim 110 \mathrm{fs}$. To avoid electrical breakdown with such high field strengths one requires more precise control of the surface quality of the photocathode, improved vacuum, and implementation of a highly reproducible, high voltage pulser for activating the photocathode. When these are achieved high brightness sources with picosecond time structures could potentially be used to illuminate sub-picosecond phenomena.

\section{Present and Future}

The table below summarizes some parameters of $4^{\text {th }}$ generation light sources important to WDM research and crucial to experiments of interest to LLNL.

There are several categories of experiments that are of interest to LLNL as a DOE/DP/NNSA laboratory. Not included here are those experiments that require high average brightness and for this discussion of greater interest are those aspects of FELs that are, roughly speaking laser like. Thus, one can consider these new light sources as lasers that will pump, probe and heat samples. The categories are:

- Creating Warm Dense matter by direct FEL irradiation

- Measuring the Equation of State of finite temperature dense matter using the FEL

- Measuring the opacity of finite temperature dense matter using the FEL as an absorption source

- Diagnosing finite temperature dense matter using the FEL (e.g., Thomson scattering or interferometry)

- Performing plasma spectroscopic experiments using the FEL as tunable laser. 
There is no fine distinction between some of these experiments as several may be appropriate at once. For example, the creation of WDM may well require the FEL to be a diagnostic also.

\begin{tabular}{|l|l|l|l|l|l|l|}
\cline { 2 - 7 } \multicolumn{1}{c|}{} & XRL & USX & SPPS & TTF & LCLS & TESLA \\
\hline mJ/pulse & 0.3 & 0.3 & 2.8 & 0.3 & 2.6 & 3.7 \\
\hline Photons/pulse & $9 \times 10^{12}$ & $3 \times 10^{2}$ & $3 \times 10^{7}$ & $9 \times 10^{12}$ & $2 \times 10^{12}$ & $2 \times 10^{13}$ \\
\hline Pulse length (fs) & 1000 & 100 & 80 & 200 & 200 & 100 \\
\hline GW & 0.006 & 3.0 & 35 & 3.0 & 26.0 & 37.0 \\
\hline Peak brightness & $1.8 \times 10^{25}$ & $6 \times 10^{19}$ & $2 \times 10^{25}$ & $2.0 \times 10^{30}$ & $1.2 \times 10^{33}$ & $8.7 \times 10^{33}$ \\
\hline Hz & $<1$ & $10^{4}$ & 30 & 50 & 100 & 50 \\
\hline Date & $(?)$ & 2003 & $2003(?)$ & 2004 & $2009(?)$ & $20011(?)$ \\
\hline
\end{tabular}

Table: Summary of the relevant light sources

In all these experiments and, in fact, in all experiment to be performed at the future light sources there will be a absolute need to a short pulse fairly intense optical laser system. This optical laser will be required to perform many tasks as central as providing a monitor of the relative jitter or providing a heating source that has a temporal structure similar to the FEL. It is this additional laser system that will provide LLNL the opportunity to become fully involved in the development of FELs as a fertile next generation light source.

\section{Overall Challenge}

It is clear that X-ray sources will play a critical role in the development of our understanding of warm dense matter. The challenge is to develop experimental techniques in the regime that utilize the advantages of these various sources. This requires a concerted effort be applied to defining a research plan that can provide data as we work toward the goal of understanding warm dense matter and at the same time perfecting techniques that lead to large-scale data generation with the accuracies required by the end users.

\section{- Energetic Materials}

\section{(J. D. Molitoris)}

The fundamental questions that we address in this overview are:

- What region of WDM phase space can be addressed by energetic materials?

- What are the compelling reasons to use this technique?

Before starting this research at the LLNL High Explosive Applications Facility (HEAF) about two years ago, we theorized that with energetic materials one could:

- Produce grams or $\mathrm{mm}^{3}$ volumes of WDM

- Create conditions which would sustain WDM for micro-seconds 
- Allow a better diagnosis of WDM with these temporal and spatial scales

- Use energetic materials as a generator and/or a driver for WDM

The primary basis for this speculation were calculations done with the Cheetah/ALE thermo-chemical/hydrodynamics code. The experiments that we have done have proven these assertions to be true. We developed an experimental technique versatile enough to perform sensitivity studies on a wide range of materials in the transition through the WDM regime. Although present work at LLNL focuses on $\mathrm{Si}$, a number of materials including $\mathrm{Al}, \mathrm{B}, \mathrm{Cu}$, and Ta can be compressed and heated. Furthermore, the technique will allow studies of ionization and opacity for microseconds. The outstanding question is to determine if thermodynamic equilibrium is maintained over this entire period. We know that WDM is transient by its very nature, but how transient? Future research will answer this. For now we have demonstrated that a phase space region from 0.3 to $\geq 1 \mathrm{eV}$ (and probably as high as $3 \mathrm{eV}$ ) in temperature and from 0.1 to $3 \mathrm{~g} / \mathrm{cc}$ in density can be covered by using energetic materials as a shock driver to compress a silica aerogel.

We started this research by examining energetic materials as a generator of WDM, not a driver. Although promising at first glance, the use of metals in energetic materials holds more promise for high temperature combustion studies than it does for warm dense matter, so we embarked on another path. By adjusting the density (porosity) of the energetic material, the calculated C-J temperature can be driven higher. This is due to the irreversible work performed by the collapse of voids in shocked porous energetic materials. Further, Cheetah calculations showed that the detonation of porous energetic materials can, in principle, reach much higher temperatures. The figure below illustrates this in a Cheetah calculation done by Fried and Howard. The temperatures are calculated along the shock Hugoniot of porous HMX as a function of solid volume fraction. Three volume fractions are shown. The smallest volume fraction (highest porosity) upon detonation yields temperatures of over $1.8 \mathrm{eV}$ and the production of a partial plasma. These calculations also showed that by varying the porosity, one could achieve a wide range of temperatures in the lower part of the WDM regime.

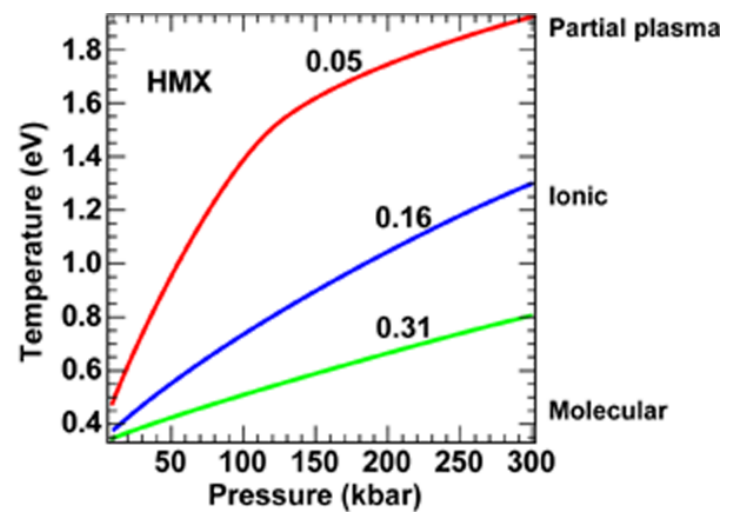

Figure: Thermo-chemical calculations done by L. Fried and W. M. Howard showing that shocked porous energetic materials can attain temperatures well into the WDM regime. Shown are calculated temperatures along the shock Hugoniot as a function of solid volume fraction. The expected state of matter is shown to the right. 
The use of porosity to generate heat became the central idea in the technique we developed. However, there are numerous complications with studying WDM in detonation. The foremost of these is the complex chemistry that takes place in a detonation and the abundance of diverse materials sustaining the detonation and forming the reaction products. It is unknown what happens just before the detonation front and just after. Even in light of these difficulties, the most severe problem is diagnosis, i.e., to see into the detonation one must use x-rays. Any emission is attenuated by the opacity of the materials involved. Because of these complexities, we resorted to $\mathrm{SiO}_{2}$ aerogels and using the energetic material as a shock driver. This arrangement solves nearly all of the problems noted.

Silica aerogel is relatively transparent to the visual part of the spectrum and radiography is possible, but difficult. Furthermore, the material is chemically simple and does not react upon shock compression. $\mathrm{SiO}_{2}$ aerogel can be produced with a range of density from less than $10 \mathrm{mg} / \mathrm{cc}$ to nearly $600 \mathrm{mg} / \mathrm{cc}$. This range of density has the ability to produce a wide range of temperatures and densities upon shock compression, thus making it extremely versatile for WDM studies. Finally, the aerogel can be seeded with a number of solid materials from boron to tantalum, thus allowing a wide range of sensitivity studies in the WDM regime. The energetic material WDM studies at HEAF have focused on silica aerogel densities of 100 and $300 \mathrm{mg} / \mathrm{cc}$ with LX-10 as the high explosive driver. Initially, single shock techniques were studied to understand the shock transmission to, and behavior in, the aerogel. As the transmitted shock dispersed in the aerogel, it cooled, thus limiting the duration of the WDM produced. The figure below is a time resolved 450 $\mathrm{keV}$ radiography sequence for a single shock WDM experiment. The time duration of each image is $60 \mathrm{~ns}$, which is sufficient to "freeze" the detonation and transmitted shock. The highest compression, density and temperature are produced right after the shock is transmitted to the aerogel as shown in image c). Here the shocked region is very thin and flat. After this, the shock front cools and decompresses in the aerogel as shown in image d) of the sequence. These data illustrate that radiography is a viable diagnostic for determining density of the WDM regions in these experiments. They also show that the temperature varies as the shock propagates in the aerogel, so there is a finite time for making a valid temperature measurement once the shock is transmitted.

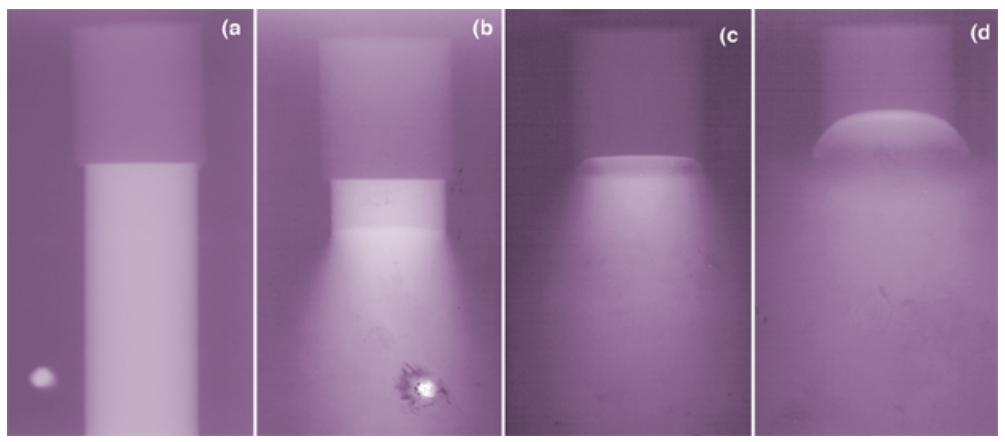

Figure: Time resolved radiography sequence of single shock WDM experiment using $450 \mathrm{keV}$ point projection imaging. 
The shock waves transmitted to the silica aerogel were sufficient to compress it to just greater than solid density. Reliable densities are difficult to extract from radiography, but not impossible. The diagnostic technique has sufficient spatial and temporal resolution, but still needs more work to reduce scattering effects that complicate absolute density measurements. Our determination of the maximum temperature produced in these single shock experiments was just above $0.6 \mathrm{eV}$. This is lower than expected, perhaps due to the long gate time in the experiment - recall that WDM is transient by its nature. Previous Russian reports of over $2 \mathrm{eV}$ produced in single shocked silica aerogels were not confirmed here. The conclusion from all available data on single shock aerogels using energetic materials is that this technique allows access to the lower temperature end of the WDM regime.

In understanding the shock behavior within these silica aerogels, we were able to design an experiment with far greater versatility for WDM studies. This experiment utilized shock drivers on both sides of the aerogel and created a sustained WDM regime by producing the highest possible densities and temperatures using colliding shocks. These colliding shock experiments are unique to the WDM research performed at the HEAF. There were no similar experiments performed anywhere else to guide us or compare results to. Our thermo-chemical/hydro calculations indicate peak pressures of greater than $1 \mathrm{Mbar}$ and compression factors (in aerogel) greater than 10 . For solid density silica the maximum compression factor is 1.13 to 1.16. Fast imaging data is shown below. This figure shows a time sequence of the experiment from detonation in a) to the production of a thin disk of WDM in d). Note that there is a clear view of the WDM filament for over a microsecond allowing time for a number of measurements to be done.

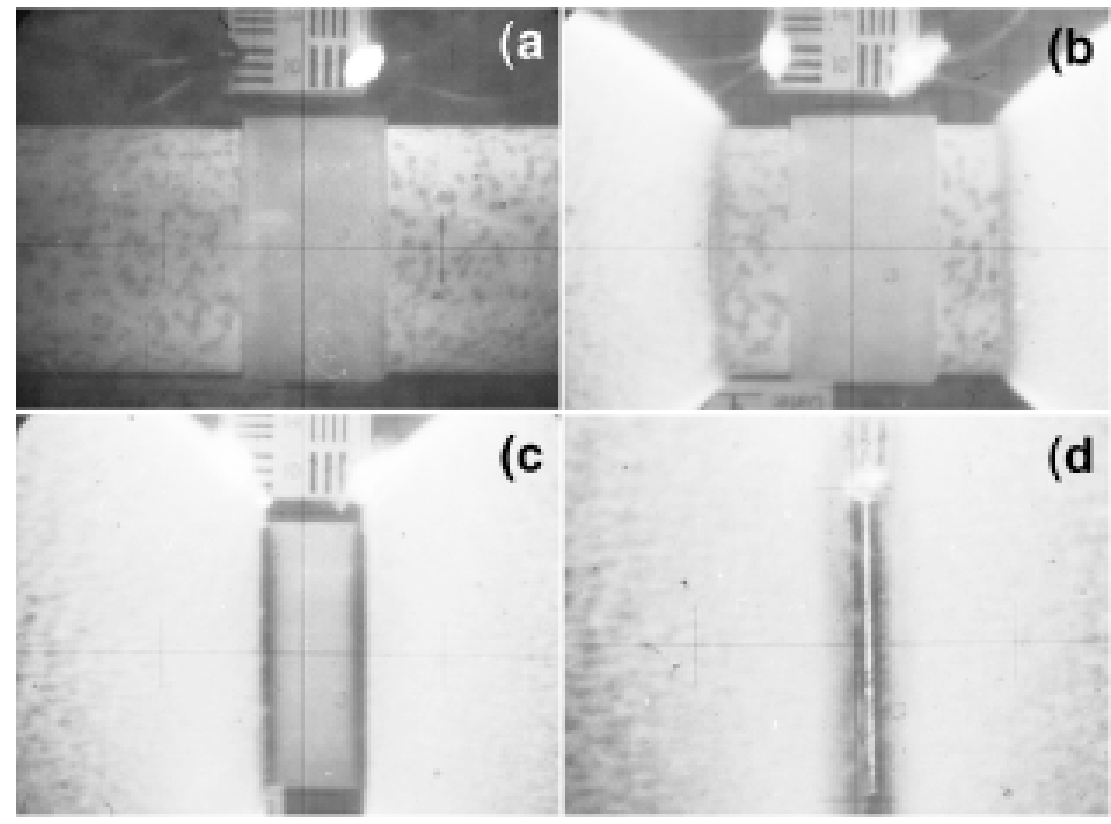

Figure: Imaging sequence of a colliding shock experiment. Sequence shows: a) Initiation (note timing fiducial); b) detonation fronts well into LX-10 driver; c) shock fronts well into aerogel WDM package; d) Production of the WDM disk. Time duration for each image is $0.42 \mu \mathrm{s}$. 
Temperature measurements using the spectral emission of silicon from the WDM region have confirmed that the temperature is well over $1 \mathrm{eV}$ for our colliding shock technique. The time resolved spectral data shows a set of constant spectral features for $\sim 1 \mu \mathrm{s}$. Experimental confirmation of the density will have to wait for detailed radiography.

These experiment allow one to estimate that the phase space accessible by using energetic materials will be up to $\leq 3 \mathrm{eV}$ and up to few times solid density. There are many compelling reasons to use this technique. The primary reason is that it has the best chance of attaining thermodynamic equilibrium and holding it for measurable periods of time. Moreover the experiment is very versatile as the aerogel can be loaded with a wide range of materials to be studied. One must only ensure to include enough material to participate in the compressive heating, but not too much to absorb a great deal of heat and drastically reduce the temperature. Finally, these experiments are self-contained. Scaled versions using less than 200 grams of material can be moved to other experimental facilities. Plans are presently underway to use the heavy-ion beams of the GSI laboratory to measure the bulk density, and potentially the electron and ion density, via ion beam energy-loss techniques. The heavy-ion density measurement will be compared to X-ray density measurements performed at the HEAF. It may also be possible to use this technique to generate WDM that can then be diagnosed using laser beams for x-ray backlighting to provide a high-resolution time sequence of the WDM and a time resolved density measurement.

\section{- Ion-Beams}

\section{(D. H. H. Hoffmann)}

Intense heavy-ion beams are an efficient tool to study the equation of state and other properties of WDM, providing new possibilities for research in WDM and high-energydensity matter. Due to the unique features of the energy deposition process of heavy ions in dense matter, and to the volumetric heating, it is possible to generate high entropy states without necessity of shock compression. Previously, such states could only be achieved by using powerful shock wave generators like nuclear explosions or powerful lasers. Here, this novel technique of heavy ion heating and expansion (HI-HEX) is proposed to explore new fascinating regions of the phase diagram including liquid phase, evaporation region with the critical point and strongly coupled plasmas (WDM).

States of matter characterized by high-energy-density occupy a broad region of the phase space diagram, including hot compressed matter, strongly coupled plasmas, hot expanded liquid, quasi-ideal plasmas and warm dense matter. Our knowledge of these states is very limited because theoretical modeling is extremely complicated and experiments are very difficult to perform. The possibilities of various experimental techniques are shown in the phase space figure for $\mathrm{Pb}$ below. This phase diagram has been calculated using a widerange multi-phase EOS [1] and the curves, indicating different experimental methods that 
have been used to investigate the thermo-physical properties of $\mathrm{Pb}$, including all the available information up to date.

The most significant information so far has been obtained using shock wave methods $[2,3,4,5]$. Due to the shock, material is compressed and irreversibly heated, leading to high pressure and entropy. Using the shock wave compression method, only a narrow region of the principal and porous Hugoniots have been investigated, see the figure below. Pressure range of up to $10 \mathrm{Mbar}$ has been investigated using high explosive generators of shock waves, light-gas guns and Z-pinches [2,6,7,8,9]. Much higher pressures in the Gbar range have been achieved in underground nuclear explosion experiments [10]. Progress in powerful lasers allows one to access the multi-megabar and Gbar regions as well $[11,12]$.

Following the ideas of Zeldovich [5] investigation of shock adiabats of porous samples extends explored region of the phase diagram to lower density and higher entropy in comparison to the principal Hugoniot [2,6,7,8]. Another idea of Zeldovich was to study expanded states of shock compressed material, i.e., the release isentropes. High entropy states along release isentropes, which originate from shocked material, occupy a region of the phase diagram below the principal Hugoniot, see the figure below. However, the amount of data that is available is limited to sets of points along a Hugoniot.

We propose to generate high entropy states in matter using quasi-isochoric heating by intense heavy ion beam. These states could be studied during the heating and expansion phases. Due to this intrinsic feature of volume heating by heavy ions in the proposed heavy ion heating and expansion (HI-HEX) technique, these exotic high entropy states can be generated without any shocks.

In such an experiment the heated material would expand, passing through different states. For example, if we start from solid density, a metal would be heated forming a hot liquid characterized by a disordered arrangement of ions and degenerate electrons. It will then expand to a quasi-non-ideal Boltzmann plasma and a rarefied vapor. Upon expansion 


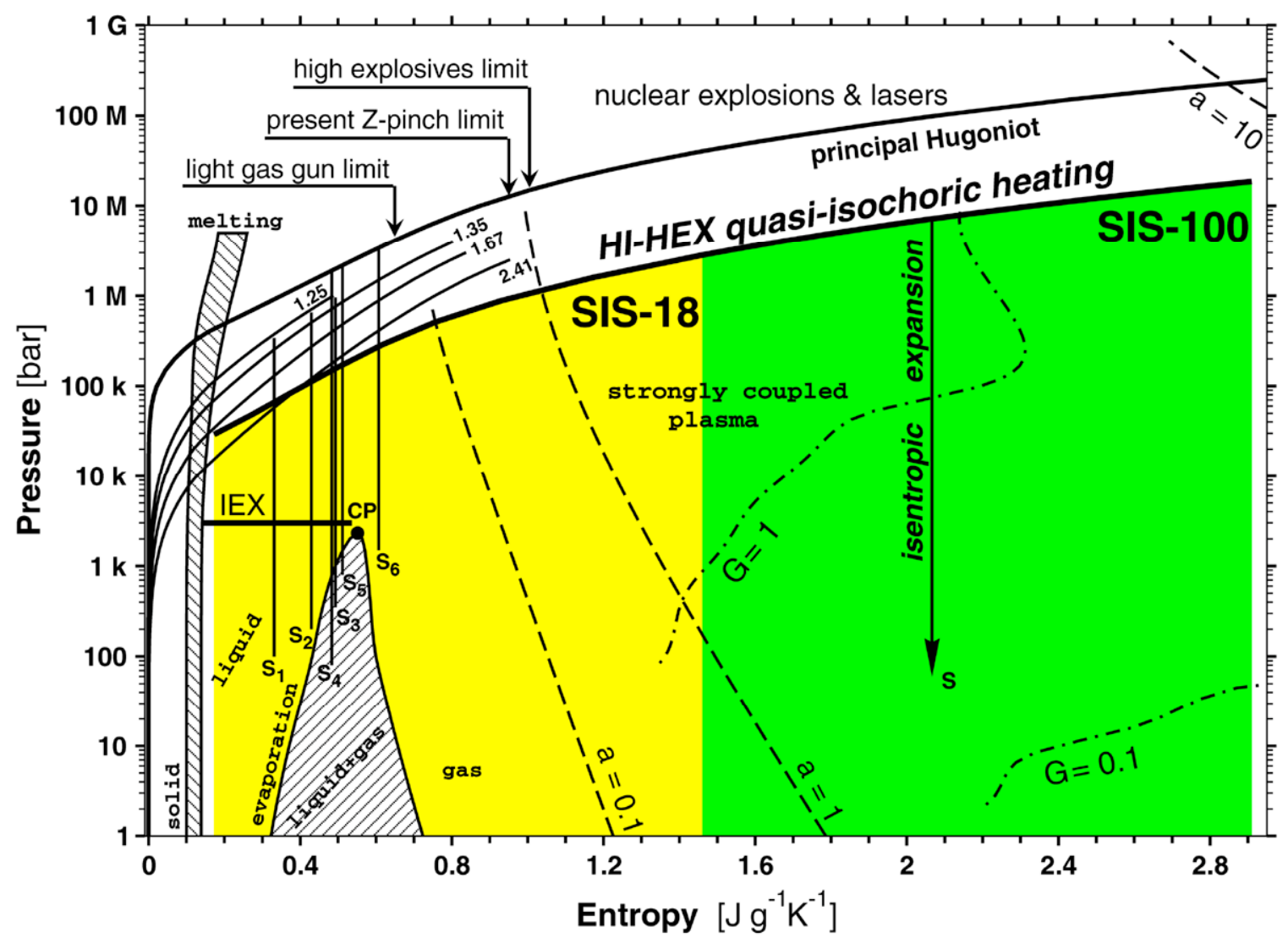

Figure: Pressure-entropy phase diagram for lead. All the experimentally studied regions using different techniques are plotted. The principal and porous Hugoniots investigated in shock wave compression experiments (the numbers near Hugoniot curves indicate porosity, $\left.V_{\text {porous }}=V_{\text {solid }}\right)$, the only studied release isentropes $\left(S_{1}\right.$ to $\left.S_{6}\right)$ from $[2,5,6,7]$ and isobaric expansion (IEX) [13] are shown along with the parameter space which will be accessible with HI-HEX technique. Possibilities of shock wave drivers are also plotted using the data from $[2,5,6,7]$ for light-gas guns, high explosive generators and for Z-pinch [8]. The physical characteristics such as melting and evaporation curves, the critical point, isolines of ionization degree (a) and non-ideality parameter $(G)$ are also shown.

of the system, the degree of degeneracy of the electronic subsystem is decreased and a marked rearrangement of the energy spectrum of atoms and ions occurs. Then a partial recombination of the dense plasma takes place. In the disordered electron system a metalto-insulator transition occurs and in isentropic expansion a non-ideal plasma (with respect to different forms of inter-particle interactions) is formed in the vicinity of the liquidvapor equilibrium curve and the critical point. When the isentropes enter the two-phase liquid-vapor region, evaporation occurs and, on the gas side, condensation occurs. If on the other hand, we induce higher entropy in the sample, more exotic states of strongly coupled plasma will be generated from the very beginning. The states on the expansion adiabat of this system will be characterized by interesting plasma effects, such as a significant variation of degree of ionization (a) and plasma non-ideality parameter (G) shown. The range of thermodynamic parameters that can be covered in a single HI-HEX experiment is extremely wide: six orders of magnitude in pressure and four orders of 
magnitude in density.

The Gesellschaft für Schwerionenforschung (GSI, Institute for Heavy-Ion Science) in Darmstadt, Germany is a unique position delivering intense beams of heavy ions from the heavy ion synchrotron SIS-18. At present this facility can provide about $10^{10}$ uranium particles with energy of a few hundred $\mathrm{MeV} / \mathrm{u}$. The pulse duration is a few hundred nanosecond. It is expected that in the near future the beam intensity will increase to $2 \mathrm{x}$ $10^{11}$ particles while the pulse duration will be reduced to less than $100 \mathrm{~ns}$. Numerical simulations have shown [14] that a beam with these parameters will deposit a specific energy of 50-100 kJ/g in solid lead. GSI also has plans to build a new synchrotron ring SIS-100 that will deliver an intense uranium beam having $10^{12}$ particles with energy range of 400-2700 MeV/u. This beam will deposit a specific energy of at least $350 \mathrm{~kJ} / \mathrm{g}$ in lead [15].

It is seen from the figure that use of these two heavy ion machines will enable us to investigate regions of hot liquid metal, strongly coupled plasmas, evaporation region with the critical point, quasi-ideal plasmas as well as the warm dense matter regime. One should note that these exotic, high-entropy, states could only be accessed previously by underground nuclear explosions. However these regions have not been investigated so far. The proposed HI-HEX method therefore opens new fascinating possibilities to study phase diagram of matter.

In our method the thermo-physical parameters like temperature, density, expansion velocity, and electrical conductivity will be measured during the heating phase and the following adiabatic expansion with time resolution. Together with numerical modeling, this information will be sufficient to determine EOS properties of the material under investigation.

Using the HI-HEX technique, with the existing SIS-18 machine one can access the region of the phase diagram that previously was accessible only by powerful nuclear explosions. Therefore the HI-HEX method is a unique and very promising tool for generation and investigation of exotic states of high energy density matter.

We wish to thank V. Gryaznov for providing calculations for lead plasma. We also would like to thank M. Kulish and members of the GSI plasma physics group for many fruitful discussions and the BMBF for providing the financial support to do this work.

\footnotetext{
1 A.V. Bushman, G.I. Kanel, A.L. Ni, V.E. Fortov, Intense Dynamic Loading of Condensed Matter (Taylor\&Francis, London 1993).

2 R.G. McQueen, S.P. Marsh, J.W. Taylor, J.N. Fritz in: High Velocity Impact

Phenomena, ed. R. Kinslow, (Academic Press, New-York, 1970) 293 and appendices

3 R.G. McQueen, S.P. Marsh, J.W. Taylor, J.N. Fritz in: High Velocity Impact

Phenomena, ed. R. Kinslow, (Academic Press, New-York, 1970) 293 4417;

appendices on pp. 515-568.

4 L.V. Altshuler, Usp. Fiz. Nauk, 85, 197 (1965) [Sov. Phys. Usp., 8, 52 (1965)].
} 
5 Ya.B. Zeldovich andYu.P. Raizer, Physics of Shock Waves and High-Temperature Hydrodynamic Phenomena (Academic Press, New York 1966, 1967)

6 M. van Thiel, ed., Compendium of Shock Wave Data, (Lawrence Livermore Laboratory Report, UCRL-50108, 1977)

7 S.P. Marsh, ed., LASL Shock Hugoniot Data, (University of California Press, Berkeley, Los Angeles, London, 1980)

8 M.V. Zhernokletov, V.N. Zubarev, R.F. Trunin, V.E. Fortov, Experimental Data on Shock Compression and Adiabatic Expansion of Condensed Materials at High Energy Density, (IPCP RAS, Chernogolovka, 1996) [in Russian]

9 M.D. Knudson, D.L. Hanson, J.E. Bailey et al., Phys. Rev. Lett., 87, 225501-1 (2001)

10 A.S. Vladimirov, N.P. Voloshin, V.N. Nogin, A.V. Petrovtsev, V.A. Simonenko, Sov. Phys. - JETP Lett., 39, 85 (1984)

11 Th. Lower, R. Zigel, K. Eidmann et al., Phys. Rev. Lett., 72, 3186 (1993)

12 R. Cauble, D.W. Phillion, T.J. Hoover, N.C. Holmes, J.D. Kilkenny, R.W. Lee, Phys. Rev. Lett., 70, 2102 (1993)

13 G.R. Gathers, Rep. Progr. Phys., 49, 341 (1986)

14 N.A. Tahir et al., Phys. Rev. E, 61, 1975 (2000)

15 N.A. Tahir et al., Phys. Plasmas, 8, 611 (2001)

\section{- Short Pulse Lasers}

\section{(K. Widmann)}

Short Pulse Lasers (SPLs) are powerful tools in WDM research as they can be used to both produce and diagnose WDM. WDM is produced by heating a target directly with the laser or by heating the target with a laser produced radiation source. The SPL is also a powerful diagnostic of WDM produced by any of the techniques discussed at this workshop (light sources/FEL, energetic materials, heavy-ions, etc.). Optical properties can be ascertained by using the SPL beam, density measurements can be made by applying interferometric techniques, and WDM can also be probed with laser produced radiation sources. 

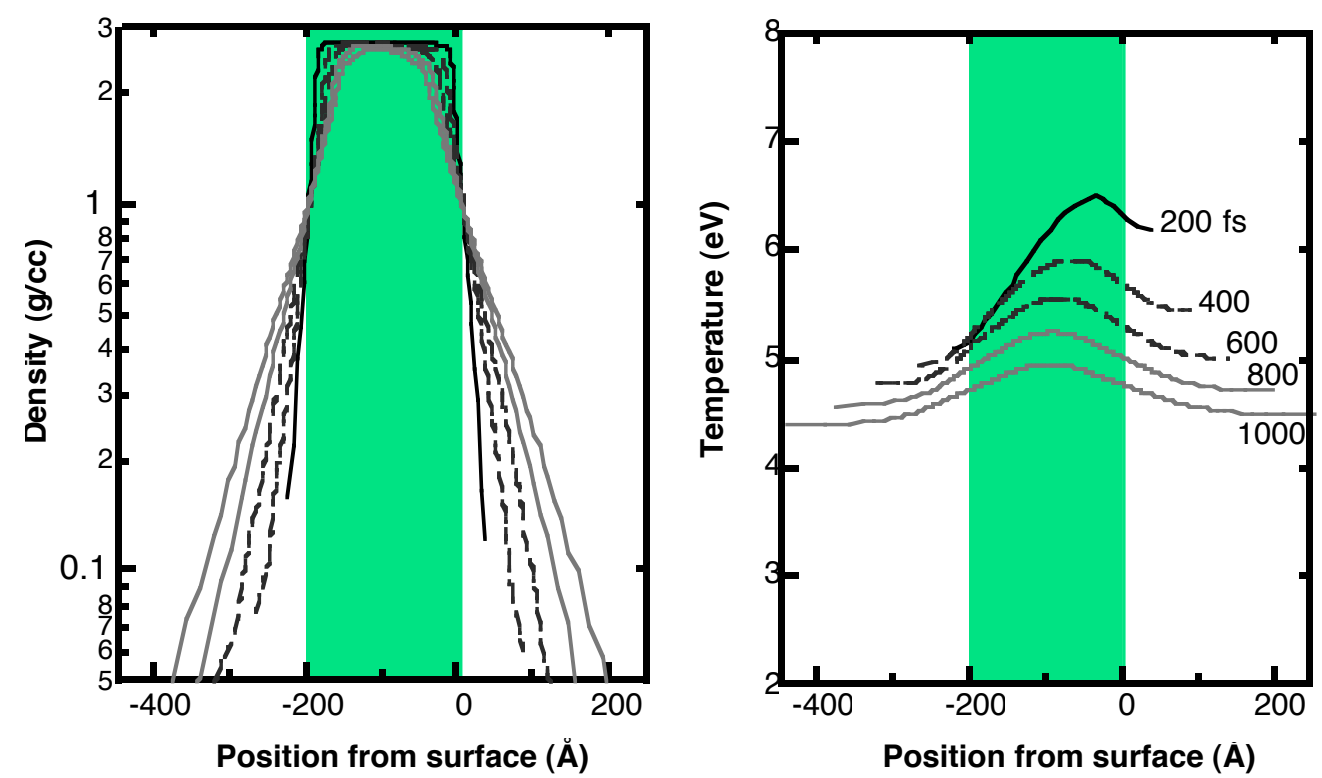

Figure: Example of achieving ISP conditions through ultra-thin target foils irradiated with a short pulse laser at $10^{13} \mathrm{~W} / \mathrm{cm}^{2}$

In combination with other techniques there is an optimistic outlook for equation of state experiments using SPLs. Contending methods that use shocks to obtain equation of state (EOS) information at the lower temperature side of the warm dense matter phase space include gas guns, local heated diamond anvil cells, energetic materials, and laser driven shocks. With current technology the gas guns and the laser heated diamond anvil cells do not reach the necessary temperatures to obtain the warm, dense regime. The situation could easily change as gas guns improve with re-shock techniques and high velocity guns becoming available. However, these are currently in the developmental stages- WDM created with energetic materials is discussed elsewhere in this meeting, but the prospect of using lasers to diagnose WDM created in this manner is tantalizing and possibilities for doing this exist at NIF and the GSI lab. On the other hand, laser produced shocks created by directly irradiating a sample, which may seem an obvious approach, have substantial problems in that the laser driver creates hot ablating plasma that preheats the sample to be shocked. This leads to the problem that the initial state of the shocked material is extremely difficult to characterize. Many methods to ameliorate this situation have been attempted. Of these, the most successful has been the work on the development of a laser driven pusher that itself does not have a preheated interface with the material being shocked. Applying this successful technique for condensed matter EOS measurements relies on larger laser facilities.

To effectively use the SPL laser to produce WDM, one needs to achieve the conditions of an idealized slab plasma (ISP). This can be accomplished by using ultra-thin target foils and appropriately lowering the laser intensity. An example of a slab-like plasma is given in the figure below. This is a comparison of the density and temperature profiles of a laser-heated 200-angstrom thin aluminum foil at the end of the 400-nm, 100-fs heating pulse for different peak intensities, approximately $200 \mathrm{fs}$ after the peak intensity of the 
pulse. The outline of an ISP is shown in the figure. The simulation on the right shows an excellent match with an ISP.

There are other ways to achieve ISP conditions. Tamped targets have been used where the material of interest is layered with coatings of carbon and $\mathrm{Si}_{3} \mathrm{~N}_{4}$ to delay the decompression associated with shock heating. The prolonged "uniformity" of a tamped target provides sufficient temporal access for fast diagnostics, such as time-resolved spectroscopy. This technique has been used in laser experiments. Finally ISP conditions can be achieved by target heating with a laser produced radiation source. This has been done by Patel and co-workers using a 100 fs USP to generate a proton pulse. As long as the thickness of the target material is smaller than the depth of the Bragg peak, the proton heating can produce a long-lived, uniform, solid density plasma. This technique has achieved ISP conditions and temperatures of $10 \mathrm{eV}$ at solid density.

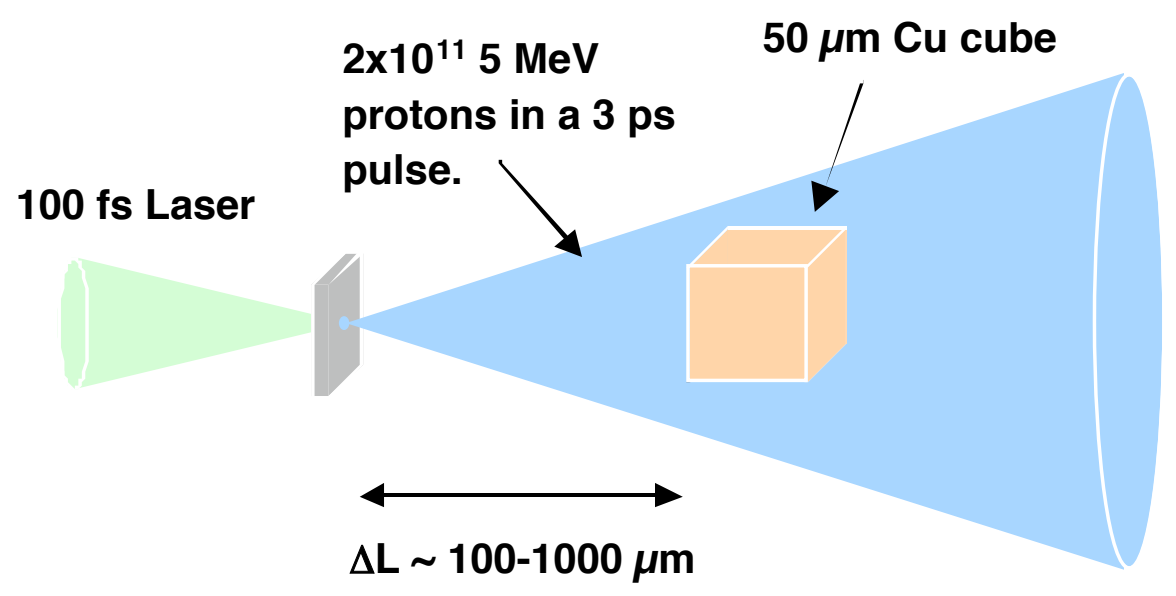

Figure: Example of using the SPL to generate a source of protons for WDM production

Using the SPL as a WDM generator allows the production of WDM at facilities extremely well suited at probing specific properties. Understanding the EOS is a good example. The experiment outlined below examines a WDM equation of state measurement proposed for the TTF facility at HASYLAB/DESY in Germany.

Equations of state of matter at high pressure (exceeding $>1$ Mbar) are fundamental to numerous applications in astrophysics, geophysics, high-pressure science, plasma physics, laboratory laser experiments, including inertial confinement fusion, and related fields. Experimentally accessing these material states offers opportunities to study phase transitions in solids near the critical point, strong coupling effects in dense plasmas, and transport properties, such as the conductivity, at high densities and temperatures. In particular, the density-temperature phase space of interest is the regime of warm dense matter, i.e., near solid densities and temperatures of a few eV up to several tens of eV. However, constraints provided by experimental results on the high pressure EOS for hot expanded states are rather limited. 
The uncertainties in the EOS can be documented by studying the uncertainties that exist between various calculations of the EOS for even the most well studied materials, e.g., $\mathrm{Al}$ and $\mathrm{Cu}$. When one compares the pressure-density-temperature (P$\rho-\mathrm{T})$ phase diagrams one finds that in the region where the temperatures are comparable to the Fermi energy large differences exist. These differences disappear, even in this phase space regime, when one is on or near the principal Hugoniot, which is the curve in the P- $\rho-\mathrm{T}$ space defined access by single shocks of various strengths. The reason the differences disappear is that along the Hugoniot experimental data exist, hence, constraining the models. In contrast, where there is no data the model deviate from each other by as much as $90 \%$. These large deviations indicate that performing experiments on the EOS are scientifically important.

High-contrast ultrashort pulse laser facilities - pulse duration in the order of 100 fs - are capable of accessing the EOS regimes of interest. Measurements of the optical properties of these rapidly heated targets have been developed far enough to yield highquality EOS-relevant data, such as the AC conductivity. The implementation of such an experiment at a short-pulse, short-wavelength laser facility - like the TTF at HASYLAB - would provide the additional tools necessary for performing EOS measurements. Indeed, the additional capability of the TTF will be a major step towards providing high quality accurate data on the EOS where previously only data related to the EOS has been obtainable in these regimes. The most relevant, and most recent, example of EOS related experiments may be found in Widmann et al. [1]

The EOS relates temperature, volume, and pressure so that an EOS measurement requires measuring two of the three state variables. In the present example we emphasize independent measurements of the density and temperature. Figure below shows a schematic of the experimental setup where a thin foil is illuminated with a SPL. The SPL is essential to heat the sample before any hydrodynamic motion takes place. The thin foil is required to achieve uniform heating of the solid [2]. The foil thickness needs to be on the order of one optical depth, i.e., approximately equal to an absorption length. Here the thinner the foil the more uniform the heating; however, the thicker the foil the longer the time before it starts to move - in the terminology of shock physics - before it unloads. In the present example we compromise the two factors using a $300 \AA$ thick foil for heating and then measuring the density and temperature. A visible SPL pulse length of $100 \mathrm{fs}$ is used to achieve heating before hydrodynamic effects take place. 


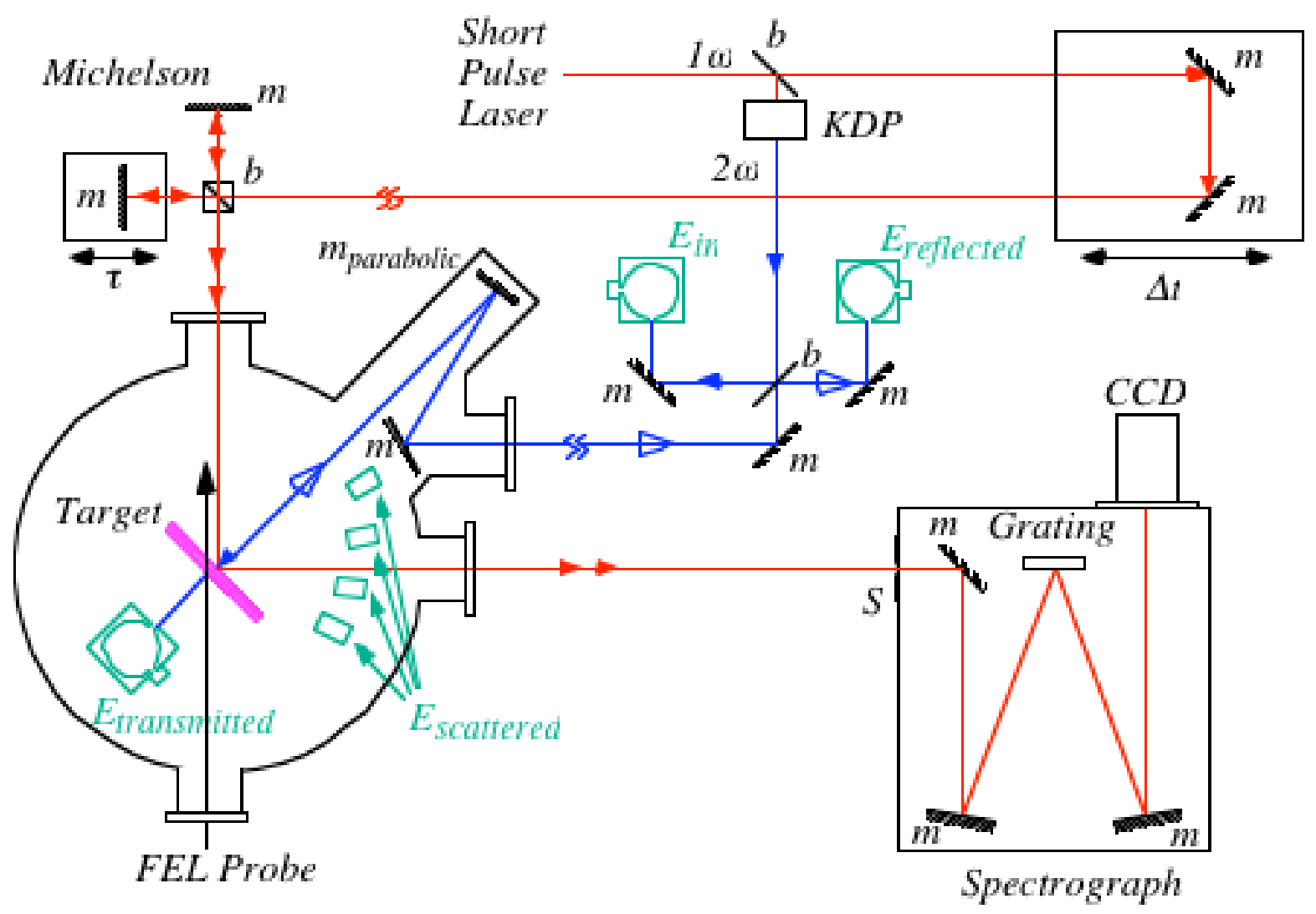

Figure. The experimental setup shows the incident optical short pulse driving laser (SPL) from the left. The laser intensity is $\sim 10^{13} \mathrm{~W} / \mathrm{cm}^{2}$ with a $100 \mathrm{fs}$ FWHM pulse length. The laser is incident on an foil of thickness $d \sim 300 \AA$. The thickness of the foil is chosen to be as large as possible while making sure that the foil is uniformly heated by the optical pulse, i.e., limiting the thickness to a single optical depth. The incident energy, reflected energy, transmitted energy, and the scattered energy are measured to determine the absorbed energy.

For the case of a $300 \AA \mathrm{Al}$ foil heated by an optical laser of intensity of between $10^{13}$ and $10^{14} \mathrm{~W} / \mathrm{cm}^{2}$ and pulse length of $100 \mathrm{fs}$ FWHM, simulations indicates an electron temperature of between $1-20 \mathrm{eV}$ in the solid density sample. In the figure below a radiation hydrodynamic simulation is shown of an optical laser with a wavelength of 400 $\mathrm{nm}$, an intensity of $10^{13} \mathrm{~W} / \mathrm{cm}^{2}$, and pulse length of $100 \mathrm{fs}$ FWHM. On the left hand side of the figure we show the time evolution of the electron temperature and the electron density of the center of the uniformly heated foil. Here the peak of the laser pulse is at $120 \mathrm{fs}$ and the maximum electron temperature is $\sim 3 \mathrm{eV}$ with an electron density of $10^{23}$ $\mathrm{cm}^{-3}$. This temperature can be seen to decrease to $\mathrm{T}_{\mathrm{e}}=1.5 \mathrm{eV}$ at $3 \mathrm{ps}$ where the electron density is seen to reduce to $10^{22} \mathrm{~cm}^{-3}$. Clearly from the time evolution indicated in the figure one needs to use a probe with time resolution of better than $500 \mathrm{fs}$. On the right hand side of the figure the electron temperature, $\mathrm{T}_{\mathrm{e}}$; ion temperature, $\mathrm{T}_{\mathrm{i}}$; and electron density, $\mathrm{n}_{\mathrm{e}}$, are shown at a time of $1 \mathrm{ps}$. The electron density indicates that the sample has a thickness of $200 \AA$ of solid density matter at this time. Here the ion and electron temperatures are essentially in equilibrium with a peak temperature of $3 \mathrm{eV}$. 

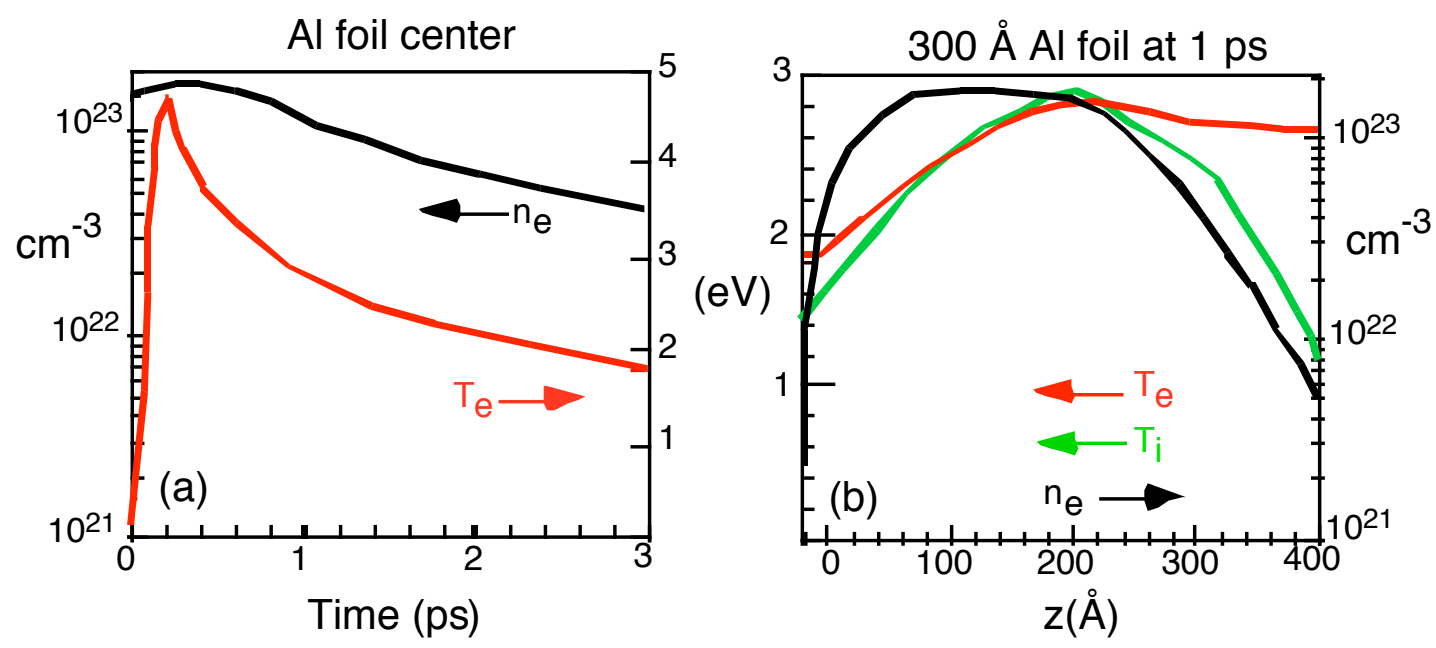

Figure: Simulations of the electron density and temperature and ion temperature for a short pulse laser-produced plasma of a thin $(300 \AA)$ Al foil. The laser has an intensity of $10^{13} \mathrm{~W} / \mathrm{cm}^{2}$ and pulse length of $100 \mathrm{fs}$ FWHM and is incident from the right hand side. (a) Shows the time evolution of the center of the foil. The peak of the driving pulse is at $\mathbf{1 2 0}$ fs and the foil remains at solid density for several hundred fs after the peak of the pulse. (b) shows the temperature and density at $t=$ $1 \mathrm{ps}$, where the ion and electron temperature come into equilibrium.

Here we would perform three measurements: the electron density using XUVinterferometry, the time-evolution of the density profile using multi-color Fourier-domain interferometry (FDI), and the electron temperature in the under-dense plasma using Thomson scattering. The first measurement uses TTF as an interferometer to measure the electron density in the solid density matter. Here a wavelength of $350 \AA$ has a critical density of $9 \times 10^{23} \mathrm{~cm}^{-3}$. The electron density of solid Al at $3 \mathrm{eV}$ is $\sim 1.7 \times 10^{23} \mathrm{~cm}^{-3}$. The electron density, $\mathrm{n}_{\mathrm{e}}$ in $\mathrm{cm}^{-3}$, is related to the measured fringe shifts as

$$
N_{\text {fringe }} \approx n_{e} L /\left(\mathrm{n}_{\mathrm{c}} \lambda\right) \text {, }
$$

where $\mathrm{L}$ is the length $(\mathrm{cm})$ of the plasma being probed by the FEL of wavelength $\lambda[3]$. For a $300 \AA$ foil at $3 \mathrm{eV}$ we should measure 0.1 fringe shift, which is easily with the observable limit and gives us the ability to measure the ionization properties of solid density matter. We can measure the electron density in a semi-conductor or an insulator to yield the ionization state of the heated solid density matter. Knowing the deposited energy density and the electron density gives us a measure of the electron temperature. In addition, FDI can provide information on the expansion velocity of the critical surface. The FDI could use a three-wavelength probe: $1 \omega, 2 \omega$, and $3 \omega$, where $1 \omega$ is the wavelength of the SPL. In this way the FDI measures the velocity of each one of three critical surfaces that by using the integral of the velocity yields the position of the critical surfaces allowing the measurement of the electron density profile.

The previous work that is relevant in this area is contained in the paper [1] and the references contained therein. Note that the TTF-FEL provides the unique possibility to measure the density of the bulk of the sample surpassing all previous measurement that have only been able to probe the surface of the expanding matter. 
Finally, we outline the use of the SPL laser as a diagnostic of WDM. The most natural use of the SPL as a diagnostic is for optical properties in the ultra-thin targets discussed above. Reflectivity and transmissivity can be determined at specific wavelengths and polarizations. Conductivity models can then be used to link transport properties to target conditions. From a single probe pulse the energy of the heating pulse can be determined, spatial profiles obtained of the pump beam spot in reflection and transmission, and a spatially resolved 2D image of the target illuminated by the probe pulse in reflection and transmission. Reflectivity data could be important in understanding the melting process and expansion of the heated target. The measurement of phase shift, reflectivity and attenuation, yield a wealth of EOS quantities. FDI, which is mentioned above, is used to precisely measure the expansion-induced phase shift. FDI can determine expansion velocity, density and temperature.

One of the most natural uses of the SPL is in point projection radiography. Short X-ray pulses generated by SPLs have been used a reliable technique to for 2D imaging and density measurements. Proton radiography is now possible with SPLs as a WDM diagnostic. In fact, the same laser can be used to produce a high-flux proton beam to isochorically heat the sample and image it with a 2D time resolved proton radiography. As proton radiography with SPLs develops it should be able to provide details on the density and materials structure of larger and thicker materials at WDM conditions.

We would like to acknowledge valuable input from J. Dunn, P. Patel, R. Shepherd, and T. Ao (UBC Vancouver) in preparation of this section.

1 K. Widmann et al.Phys. Plasmas 8, 3869 (2001).

2. A. Forsman, et al, Phys. Rev. E 58, R1248 (1998)

3 H.-J. Kunze, in Plasma Diagnostics, edited by W. Lochte-Holtgreven (North-Holland, Amsterdam, 1968)

\section{- High-Energy Density Lasers}

\section{(N. Landen and D. Kalantar)}

High energy lasers are a flexible tool for studying different states of matter. Laser pulse shaping used in combination with a range of different driver configurations allows access to a wide range of the phase space described as WDM. Specifically, techniques have been developed on high energy lasers to compress and heat materials under isochoric, isobaric, isothermal, impulsive, or isentropic processes. Gigabar pressures are attainable by shock heating and $\mathrm{PW} / \mathrm{g}$ and $\mathrm{MJ} / \mathrm{g}$ power and energy deposition levels are achievable by volumetric heating using hard x-rays generated from laser plasmas. As a result, a wide range of physics issues can be tackled using high-energy lasers, ranging from phase changes at the lowest temperatures to ionization balance at the higher temperatures. We discuss both the flexibility of high energy lasers in generating WDM conditions, and the application of these lasers for diagnosis of WDM. 


\section{- Methods to Heat and Compress mater}

We first describe different methods of heating and compressing materials are used to access the range of temperature and pressure states:

- Strong shock:

Direct laser or X-ray irradiation of a low-Z ablation layer is used to launch strong shocks. Single shock pressures up to Gbar may be achieved. This accesses states along the single-shock Hugoniot. In this case, the material typically exceeds the melt temperature under compression. An example of the high pressure that can be achieved using a single shock generated with a high intensity laser is described in by Cauble et al. [1]

\section{- Multiple shocks:}

Laser pulse shaping may be used to generate a sequence of time-delayed shocks by direct laser or $\mathrm{x}$-ray irradiation of a low-Z ablation layer. These separate shocks may be used to compress a sample nearly isentropically to $10 \mathrm{~s}$ of Mbar at low temperature $(<1 \mathrm{eV})$. Experiments have been done on the Nova laser to compress an Al foil at a peak pressure of $1.8 \mathrm{Mbar}$ nearly isentropically. To achieve this, a sequence of 3 shocks was used to bring the foil up to high pressure below the melt curve. [2] A calculation of the temperature of the compressed $\mathrm{Al}$ as a function of compression is shown for this experiment in the figure below. By using a series of shocks, the foil is compressed nearly isentropically.

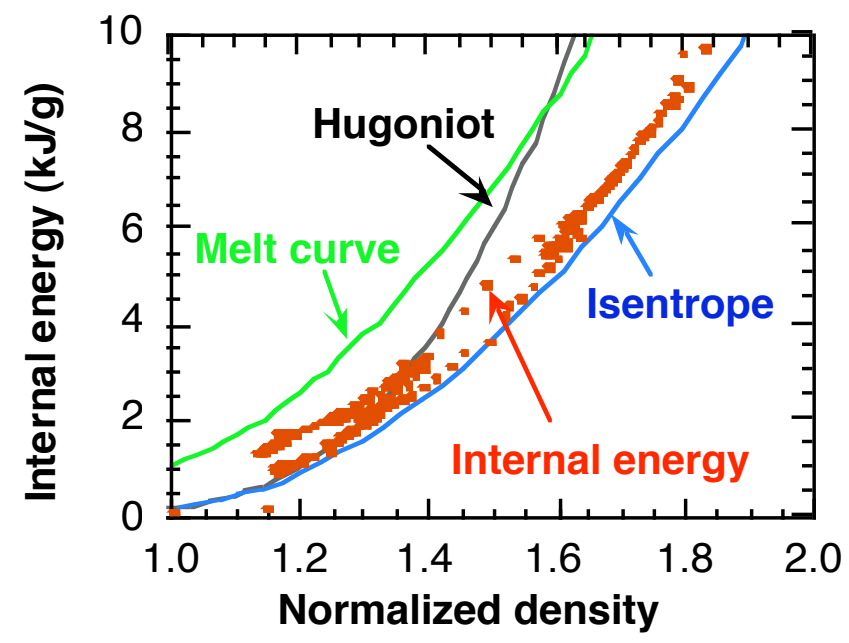

Figure: Internal energy plot showing near-isentropic compression can be achieved with a multiple shock drive using careful pulse shaping with a large laser

- Flyer plate:

A foil may be accelerated by direct laser or x-ray irradiation. This may then be used as an impactor to generate a single shock at high pressure. Pressures in excess of $1 \mathrm{Mbar}$ 
may be achieved with a very thin flier. The flier plate geometry is similar to the impactor configuration on a gas gun, but the sizes and time-scales that can be achieved are much smaller. This is typically a risky approach since the process of accelerating the flier plate results in instability growth of pre-existing modulations in the flier and modulations impacted by a non-uniform intensity profile of the ablation source (laser or x-ray drive).

- $\underline{\text { Isentropic drive: }}$

A shockless drive is generated using a high intensity laser by launching a strong shock in a low density foam. The foam unloads across a vacuum gap and accumulates against the sample material. The material pressure increases with a ramp to pressures up to 10 s of Mbar. This approach to compression for materials is currently being evaluated with OMEGA experiments. A series of tests have been done to compress $20 \mu \mathrm{m} \mathrm{Al}$ foils up to several hundred kbar. This is achieved with a $7 \mathrm{~ns}$ laser square laser pulse incident on a thick foam layer. The foam releases across a vacuum gap, and generates a ramped material pressure as it accumulates against the thin $\mathrm{Al}$ foil. Peak pressures in excess of 500 kbar may be achieved. [3]

\section{- Isochoric heating:}

High fluxes of x-rays and protons may be generated by short and long pulse laser-matter interactions. Heating of materials has been demonstrated using samples as large as 500 $\mu \mathrm{m}$ in size for $\mathrm{ns} \mathrm{x}$-ray sources and $50 \mu \mathrm{m}$ in size for ps high energy proton sources. In these examples, the energy deposition occurs volumetrically. Experiments at OMEGA have isochorically heated $500 \mu \mathrm{m}$ diameter solid Be and $0.8 \mathrm{~g} / \mathrm{cm}^{3} \mathrm{C}$ foam samples up to $50 \mathrm{eV}$. [4] This is achieved by $1 \mathrm{~ns}$ duration laser irradiation of a mid- $\mathrm{Z}$ overcoat to produce copious amounts of L-shell 2-4 keV radiation with a mean-free path comparable to the sample size. Simulations show temperature uniformity of $\pm 10 \%$ is achievable, and the sample can be probed for 2 ns before significant hydrodynamic motion occurs. Experiments have also been done using an USP laser to demonstrate isochoric heating using a high flux of protons. This is described in the previous section. In this case, the short pulse (100 fs) laser was used to generate a high flux of protons. A $50 \mu \mathrm{m}$ cube sample of material was heated volumetrically with these protons to $10 \mathrm{eV}$ at solid density.

\section{- Implosions:}

Integrated experiments involving multiple shock loading and isentropic compression of a spherical capsule place the capsule fuel material into a high density, high temperature state at $10^{25} \mathrm{~g} / \mathrm{cm}^{3}$ and $100 \mathrm{eV}$. Inertial confinement fusion targets are designed to implode a DT-filled capsule to create temperature-density conditions sufficient to generate neutrons but fusion reactions. The point design NIF ignition capsule employs a Br-doped $\mathrm{CH}$ capsule that is driven with a series of four converging shocks. [5] The shocks approximate an isentropic compression, overlapping inside the inner surface of the capsule. The DT fuel is compressed on deceleration to achieve fusion conditions. 


\section{- Bright X-ray Sources Creation}

Next, we describe techniques to use high-energy lasers to create bright x-ray sources. With multiple laser beams, laser-based experiments can be done over a wide parameter space, while also providing diagnostic capability. Some of the diagnostic techniques that have been demonstrated with high intensity lasers are discussed below.

\section{- $\underline{X-r a y ~ r a d i o g r a p h y:}$}

High intensity $\mathrm{x}$-rays are used to radiograph target samples. This technique is used to measure the growth of pre-imposed Rayleigh-Taylor unstable perturbations under acceleration by face-on radiography. This is also useful to record the motion of a foil as it is accelerated by direct laser or x-ray irradiation, or to record the evolution of a shock interacting with a buried feature in a shock tube.

\section{- Absorption spectroscopy:}

The absorption of a compressed/heated material may be recorded using a continuum xray source such as an L-shell or M-shell emission spectrum or the Bremsstrahlung spectrum from an implosion capsule. Absorption spectroscopy may be used to study spectroscopic structure of a highly ionized plasma to characterize the pressure and density of the plasma. It also may be used to characterize the temperature and density of a solid sample in the case of extended x-ray absorption fine structure spectroscopy (EXAFS). EXAFS spectroscopy provides information on the inter-atomic spacing and degree of order in a solid. Modulation of the absorption frequency and amplitude occur near an absorption edge are characteristic of the lattice order. [6] This technique is being used to characterize the state of a Ti foil that is shock compressed by direct laser irradiation. The spectrum of emission from a direct drive implosion capsule near $5 \mathrm{keV}$ is recorded through the thin Ti foil. There is a frequency modulation in the absorption spectrum near the K-absorption edge of Ti due to the lattice order in the Ti. This technique provides information on the inter-ionic spacing and degree of disorder at temperatures as low as $0.07 \mathrm{eV}$. [7]

\section{- Bragg diffraction:}

Bright sources of x-rays are used to probe the lattice structure of a material under shock compression. X-rays diffracted from the material are recorded with angular resolution on static film or time-resolved X-ray streak cameras. This technique has been used to observe 1D vs 3D compression of a lattice as it responds to dynamic loading. This technique of in-situ dynamic Bragg diffraction was demonstrated on Nova and OMEGA for shock compressed single crystals of $\mathrm{Si}$ and $\mathrm{Cu}$. A sample image from $\mathrm{Si}$ is shown below. Here, X-rays with a wavelength of $1.85 \AA$ created by a laser beam incident on a thin Fe foil were diffracted from the (400) lattice planes of a Si crystal. The X-rays were diffracted at the Bragg angle corresponding to both the uncompressed lattice and the compressed lattice spacing as the shock propagated through the sample. The position 
along the detector corresponds to the angle of diffracted $\mathrm{x}$-rays, providing a direct measurement of the lattice spacing present in the crystal during shock loading. [8,9]
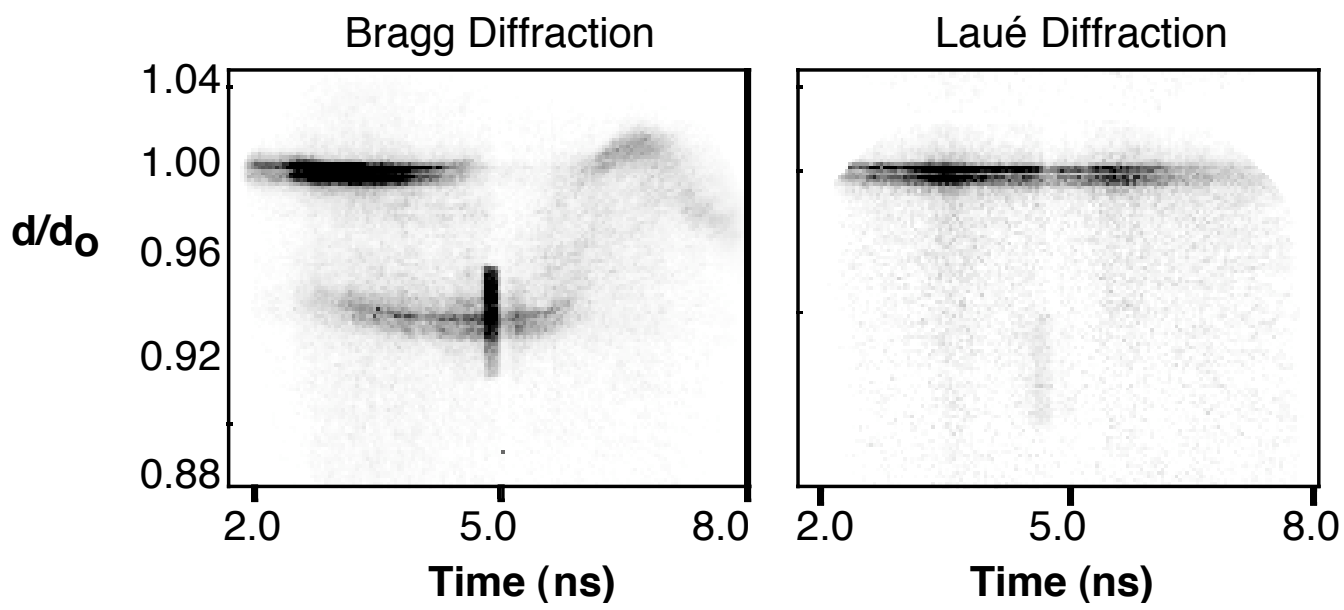

Figure: Example of in situ dynamic x-ray diffraction from multiple lattice planes of single crystal Si during shock loading. The ordinate is the normalized lattice spacing $d / d_{o}$, The left hand side shows the Bragg diffraction signal indicating compression along the shock direction. The right hand side show the Laué diffraction signal indicating no compression orthogonal to the shock direction.

- X-ray scattering:

Coherent scattering from $\mathrm{x}$-ray line sources generated by a laser-produced plasma has been demonstrated for measuring the ion-ion structure factor $S_{\mathrm{ii}}(\mathrm{k})$ and inferring the ion temperature. [10] By spectrally resolving the scattered x-rays, incoherent Thomson scattering provides information on the electron temperature density. [8] The technique of $\mathrm{x}$-ray Thomson scattering has been demonstrated using the OMEGA laser to preheat a $\mathrm{Be}$ target using $\mathrm{x}$-rays generated by direct laser irradiation of a thin Mo overcoat. A separate source of line $\mathrm{x}$-rays is created with a Ti foil, that scatters from the high density Be target. A Compton downshifted and Doppler broadened Thomson scattered $\mathrm{x}$-ray signal was recorded. [11]

- X-ray opacity:

A soft X-ray laser as been used to measure the opacity changes in compressed Al heated to approximately $1 \mathrm{eV}$. In this case, the increase in opacity is attributed to a transition to a liquid metal phase. [12]

- VISAR:

Velocimetry techniques are used to characterize the wave profile on shock breakout. This provides information on the equation of state and material properties of samples. One example of an EOS measurement is the work with cryogenic deuterium. Shock 
Hugoniot measurements were made on Nova at pressures up to 1 Mbar. [13] These results are critical for tuning the shocks in an ignition target.

The NIF laser will extend by an order of magnitude either the accessible pressure (energy density) or the experiment duration and/or size over current laser facility capabilities. NIF will hence access new regimes and improve measurement accuracy. Several NIF experiments involving warm, dense matter have already been proposed and partially designed. Four examples are isentropic compression measurements over a range of strain rates, EOS measurements in the 0.1-1 Gbar regime where compressibility depends on the ionization balance model assumed, X-ray Thomson scattering for sensing the adiabat of the imploding low Z fuel in implosions [14], and simultaneous measurement of the pressure and ionization state of a shock compressed material $(\mathrm{Ge})$ through absorption spectroscopy [15] as a means of distinguishing between EOS models [16].

High-energy density lasers are large complex facilities that have a great deal of infrastructure to allow complex experiments to be performed. Nova, the Omega laser, and in the future NIF allow experimenters to perform a class of experiments that could not be done anywhere else. The combination of complex targets with multiple drive beams, multiple backlighter/diagnostic beams, and unique well-maintained diagnostics allow unique experiments. We finish with a schematic figure to illustrate this.

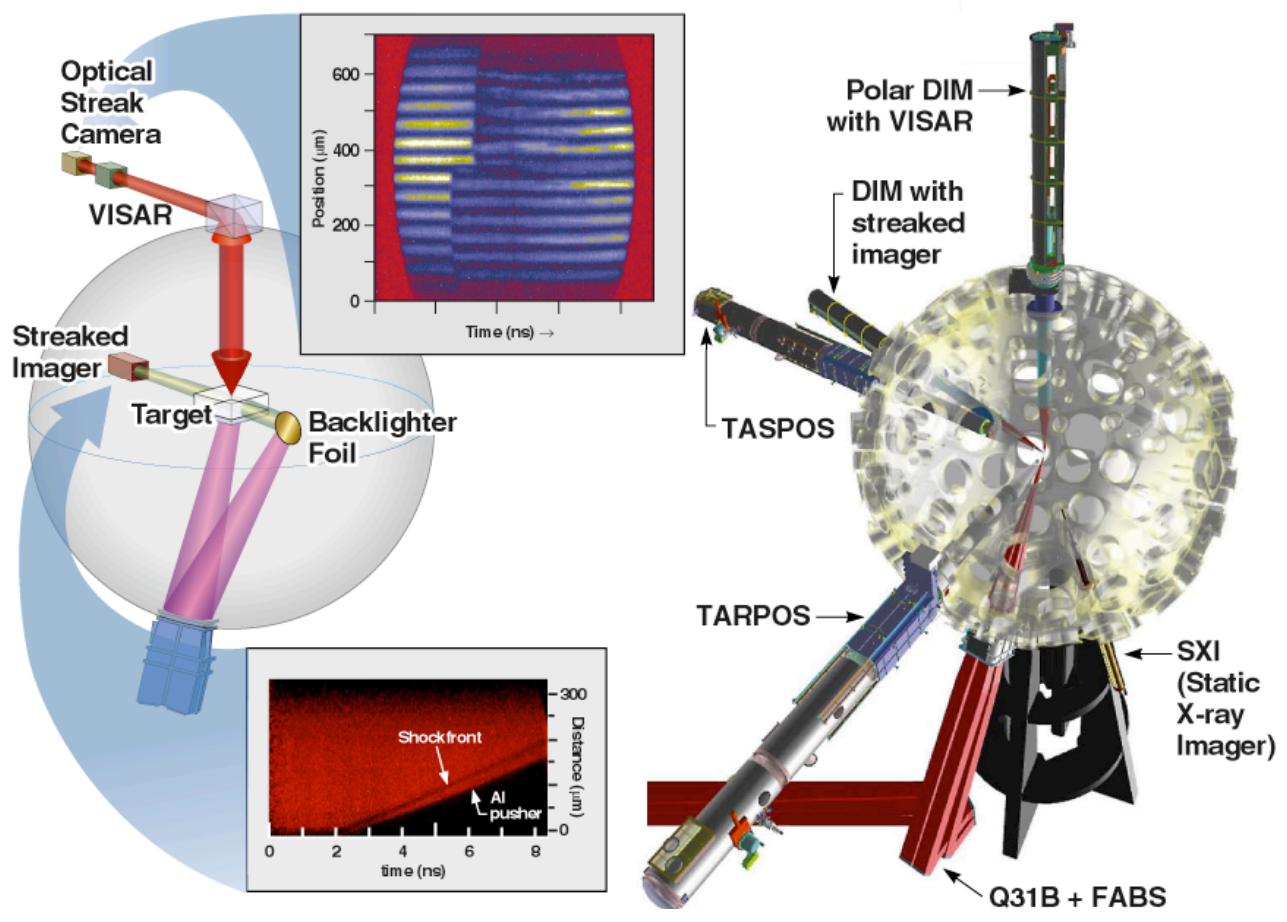

1 R. Cauble, D. W. Phillion, T. J. Hoover, et al, Phys. Rev. Lett 70 (1993) 2102.

2 D. H. Kalantar et al, Phys. Plasmas 7, (2000) 1999

$3 \mathrm{~J}$. Edwards, et al., in preparation. 
4 S. H. Glenzer, et a.l, in preparation.

5 S. W. Haan et a.l, Phys. Plasmas, 2 (1995) 2480

6 D. K. Bradley et al., Phys. Rev. Lett. 59 (1987) 2995.

7 B. Yaakobi et al, Phys. Plasmas 4 (1997) 3021

8 D. H. Kalantar et al, Rev. Sci. Instrum. 70 (1999) 629

9 A. Loveridge-Smith et al, Phys. Rev. Lett. 86 (2001) 2349

10 D. Riley et al., Phys. Rev. Lett. 84 (2000) 1704

11 O.L. Landen et al., J.Q.S.R.T. 71 (2001) 465

12 E. Wolfrum et al, J. Phys. B 34 (2001) L565

13 P.M. Celliers et al, Appl. Phys. Lett. 73 (1998) 1320

14 J. Lindl, Phys. Plasmas 2 (1995) 3933

15 T.S. Perry et al., Phys. Rev. E 54 (1996) 5617

16 D. Liberman and J. Albritton, J.Q.R.S.T.. 51 (1994) 197

\section{- Theoretical Review}

The discussion on theoretical developments for the warm dense matter regime was freeranging with many individuals contributing their opinions. Although there were numerous positions discussed, the main topics were the difficulties in getting sufficiently rigorous measurement in the regime that could assist the theoretical developments. This discussion eventual led to an effort to define a set of experiments that could be used to provide data that the theorists would accept as valid. Most of that discussion did not elaborate on the plans of individuals to develop techniques to approach the WDM regime. Below we provide brief synopses of the various efforts at moving into the WDM regime, with those attacking the condensed matter end of the WDM regime first, followed by those focused on the plasma end next.

\section{- Average Ion Approach}

\section{(J. Albritton)}

Here we describe the work in using and improving the INFERNO electron equation-ofstate (EOS) model of Liberman [1]. The model is characterized as a relativistic quantummechanical, QM, thermal average-atom/atomic-sphere/muffin-tin/atom-in-jellium model. In this method the exchange-correlation energy takes a density-functional form and all electron energy states are occupied according to the Fermi-Dirac distribution. The nuclear charge, mass density or Wigner-Seitz sphere radius, and temperature are input, and the chemical-potential that produces electrical neutrality of the atomic-sphere is determined. The potential inside atomic-sphere is the standard self-consistent-field, and the potential outside the sphere is uniform, hence the muffin-tin characterization, and the interior wave-functions for both the discrete and continuous spectrum are matched onto freewaves at the sphere-radius. The discrete spectrum wavefunctions are normalized to unity in the infinite domain, and the continuous spectrum wavefunctions are similarly energy 
normalized and described via phase-shifts. A positive ion charge density, a jellium, exists outside the atomic sphere and neutralizes the infinite domain.

INFERNO is the direct improvement of the semi-classical, SC, thermal Thomas-Fermi model of Feynman [2], with the sum of the squares of the QM wavefunctions replacing the SC density-of-states for both the bound and free electrons, and thereby also in the associated contributions to the number, energy, and entropy of the system.

The QM atomic-sphere model obeys a pressure theorem that is similar to the band model (see Ross [3]) and the SC model. The difference arises from the fact that there are residual corrections, often small, resulting from the fact that the QM atomic-sphere model wavefunctions are normalized in the infinite domain and not the finite domain of the atomic-sphere or corresponding unit-cell of a band model. Indeed, the QM atomic-sphere model gives a modestly accurate cold-curve EOS for many elemental materials.

For many elemental materials the QM atomic-sphere model yields a "softer" EOS, i.e., predicts more compression at the same pressure, than the SC model in the region of maximum compression by a single shock. This is similar to the results recently reported for the calculation of the principal Hugoniot of aluminum for example, by Rozsynai [4]. Further, and importantly, the QM model exhibits "shell-structure" effects in this region .

The QM atomic-sphere model naturally yields the solution of the electron-ion elastic scattering problem, that is, the distorted-wave differential scattering cross-section for the electron-screened nuclear charge. Then the coefficients of electrical and thermal conductivity, electron-ion energy equilibration, and so on, may be calculated according to the Ziman/Boltzmann treatment, see for example Hansen and McDonald [5]. In the regime of warm-dense-matter these calculations naturally involve the contribution of ion correlations, and we have the electron-screened nuclear charge potential from the QM atomic-sphere model for use in the ion-ion interaction calculation.

Of course, the most important work to be done is the design and replication of experiments in the otherwise almost inaccessible regime of warm-dense-matter. To this end we are also pursuing the connection of our QM atomic-sphere model to thermal band-models, quantum-molecular-dynamics models, and other independent firstprinciples descriptions

1 D. A. Liberman, Phys. Rev. B, 20, 4981 (1979)

2 R. P. Feynman, N. Metropolis, and E. Teller, Phys. Rev. 75, 1561 (1949)

3 M. Ross, Phys. Rev. B 179, 612 (1969)

4 B. F. Rozsnyai et al., Phys. Lett. A 291, 226 (2001)

5 J. P. Hansen and I. R. McDonald, Theory of Simple Liquids, Academic Press (1986) 


\section{- Warm Dense Matter from First-Principles Simulations}

\section{(G. Galli)}

The Quantum Simulation Group at Lawrence Livermore National Laboratory has developed and applied $a b$ initio techniques to study matter under extreme conditions, i.e., high temperature and pressure. These ab initio approaches do not require input from experiments and they provide predictive tools to study matter at the microscopic level. Predictive simulations are particularly important for the understanding of hot, compressed fluids where the basic constituent species are unknown and many complex phenomena, including molecular dissociation, ionization, and electronic excitations may play key roles.

There are two main techniques that have been used to study hot, compressed matter. These are the ab initio Molecular Dynamics (MD) and Path Integral Monte Carlo (PIMC). The former is appropriate and accurate for temperatures up to $\sim 2 \mathrm{eV}$ and pressure up to tens of Mbars; while the latter is accurate and computationally affordable at much higher temperature, in the same pressure range. However, PIMC has been applied only to simulations of hydrogen. If PIMC is extended to elements heavier than hydrogen and coupled to the $a b$ initio MD calculations, then a first principles simulation method would be available to investigate the structural, electronic, and vibrational properties of warm dense matter (see figure below).

The theoretical and technical challenges involved in the extension of PIMC and in the coupling with $a b$ initio MD are rather formidable. Nonetheless, the intention is to tackle these problems, since the appropriate combination of expertise exists in both techniques: PIMC and $a b$ initio MD. Not only do the combination of individual researchers exist, but they actively working at optimizing computer codes to best apply these techniques on LLNL ASCI computers.

The Warm Dense Matter (WDM) Regime is important to the understanding of many problems relevant to the success of, e.g., NIF, including target fabrication and the interpretation of the many laser shock experiments that NIF will enable. Therefore, developing techniques to study WDM is an excellent opportunity to contribute directly to the NNSA program.

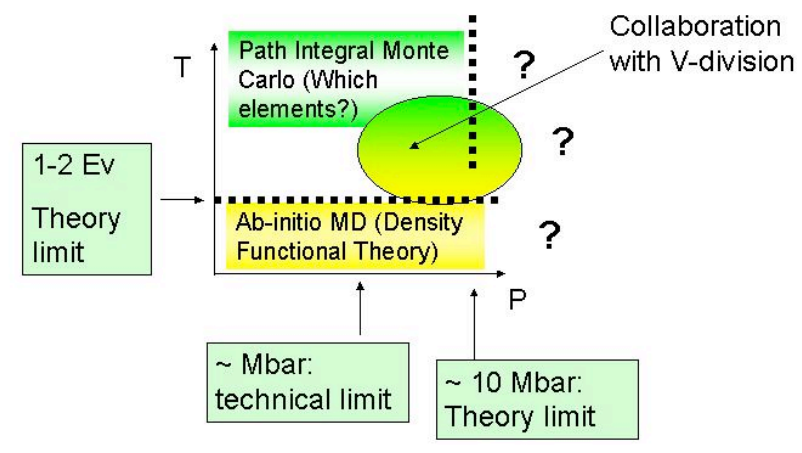

Figure: Range of validity of current first-principles molecular dynamics techniques 


\section{- Equation of State Model for Condensed Matter}

\section{(M. Surh)}

Recently, ab initio, plane wave molecular dynamics calculations have been adapted at LLNL to treat dense plasmas for temperatures exceeding the electronic Fermi temperature, TF. This approach has the advantage of conventional condensed matter theories in that the electrons are treated fully quantum mechanically (in the local density approximation, LDA), and that sampling of the ionic degrees of freedom by molecular dynamics automatically includes the effect of ion-ion interactions to all orders. This selfconsistent method also ensures that the electron-ion Coulomb interaction is incorporated non-perturbatively. Thus, the approach circumvents many of the difficulties of conventional plasma theories in the strong-coupling or semi-degenerate regimes. This new method is applicable from temperatures of $100 \mathrm{eV}$ down to the ground state, where it reduces to conventional condensed matter calculations. The initial application, to the aluminum Hugoniot, has been highly successful [1].

As it exists now, there are several limitations to this approach. The ab initio molecular dynamics method is computationally very demanding at high temperatures. In practical applications, this restricts the calculations to small systems with limited sampling and larger statistical errors. The presence of thermally excited ion cores is also treated by approximation with final state pseudopotentials. This makes it especially difficult to deal with continuum lowering and core-core overlaps, as occur at the highest temperatures and pressures. The existing pseudopotential treatment of the core electrons is also not fully consistent with the valence and conduction states.

There are specific remedies to the existing computational approach that could be explored and implemented with a concerted effort. They offer the potential to develop a computational scheme of broad utility in the regime of warm dense matter. The plan to improve the theoretical EOS of dense plasmas includes multiple avenues to a workable computational scheme.

First, an INFERNO-like approach should be taken with the pseudopotential generation. It would then be possible to iterate the single-site, spherically-symmetric pseudopotential generation with the molecular dynamics simulation of the local environment to achieve a self-consistent estimate of the core configuration for a given temperature and density. This will permit the inclusion of continuum lowering effects and allow for the possibility of theoretical plasma transitions of the average core state.

Second, the efficiency of the molecular dynamics calculation must be increased. Currently, the calculation of a Hugoniot is possible for elements such as copper or lead, but a reasonable sampling of the full phase diagram is impractical. Furthermore, the small system size required at high temperatures prevents the direct study of chemical mixtures. An obvious remedy is to create a parallel-processing version of the existing computer 
code. There is an additional possibility of using a so-called divide and conquer scheme to partition a large system into more manageable fragments.

This approach should be viable at temperatures on the order of the Fermi temperature because the electronic density matrix becomes short-ranged there. The divide and conquer method may also make it possible to include core electrons explicitly in the calculation. The scheme then begins to resemble a multi-site, non-spherically-symmetric version of INFERNO. This presents exciting possibilities for dense plasma calculations.

Finally, the information generated by the molecular dynamics scheme should be put to full use. The basic procedure uses molecular dynamics to integrate over phase space and produce an equilibrium distribution. Along the way, it generates instantaneous atomic configurations and a full spectrum of electronic wavefunctions. Molecular dynamics calculations on larger systems with better statistics will permit the calculation of sound speeds and dynamic structure factors, $S(k, \omega)$. Non-equilibrium quantities such as conductivity or optical properties can be estimated from the electronic wavefunctions generated during the calculation.

This suggests the following objectives for improving the EOS theory for warm dense matter. First, we will implement a fully-relativistic pseudopotential generation code for heavier atoms in a plasma environment. We can also examine strategies to parallelize the existing molecular dynamics code and to partition large domains into smaller subsystems. Exploratory work is necessary for the divide and conquer approach, because the equilibration of the electron chemical potential between different domains may be difficult to achieve efficiently. Calculations for the smaller subsystems would still be extremely time-consuming, but the approach is trivially parallelizable, by subsystem.

Second, we will ascertain the suitability of divide and conquer methods for dense plasma simulations. We will examine all-electron estimates of core-core overlap parameters in a dense plasma, and develop pair-potential or tight-binding corrections to the ion-ion interaction. We will combine the plasma atomic pseudopotential calculations with the molecular dynamics equation of state to look for plasma phase transitions in the atomic core configuration.

Third, we will incorporate the core-overlap effects as corrections to the earlier theoretical EOS and directly in further molecular dynamics simulations. We will combine the plasma atomic pseudopotential with a detailed molecular dynamics treatment of the ionion pair correlation function to self-consistently determine the electronic core configuration of an atom in a dense plasma. We will extend the aluminum molecular dynamics calculations to estimate sound speeds along the Hugoniot. Finally, we will examine the EOS for a heavy element, such as lead. If the divide and conquer approach has proven to be feasible, we will apply it to the $\mathrm{Pb}$ EOS, or to a similar system.

1 M. P. Surh, T. W. Barbee III, and L. H. Yang, Phys. Rev. Lett. 86, 5958 (2001) 


\section{-WDM Equation of State from the Plasma Point of View}

\section{(F. Rogers)}

The warm matter regime poses difficulties for plasma theories because standard methods based on plasma physics are not optimal for normal density material heated to a few eV; i.e., relevant expansion parameters are $\geq 1$. As a consequence EOS tables are constructed using interpolation methods to connect the condensed matter and plasma regimes. Even though the physical properties of this region are important to a number of laboratory applications the adequacy of this procedure has largely gone untested by experiment. In view of this fact it is important to undertake a program to improve and extend the plasma theoretic models down toward the Fermi temperature, $\mathrm{T}_{\mathrm{f}}$.

The EOS of dense plasmas from first principles methods has proven difficult. There are two main reasons for this: 1) description of plasma effects on bound states, especially in the region of pressure-ionization, and 2) description of Coulomb interaction effects on the plasma structure. This situation has led to the adoption of ad hoc methods that are difficult to extend. Here we will pursue the activity expansion method (ACTEX) developed at LLNL, which is an alternative to ad hoc methods. On the one hand, the classical "Coulomb catastrophe" associated with the attractive electron-ion interaction makes this a quantal problem; however, many-body quantum calculations can only use a few low-order terms in the quantum-mechanical activity expansion. On the other hand, the ACTEX method is able to resolve this by using a complete activity expansion.

Currently ACTEX includes the quantum-mechanical electron-electron and electron-ion terms to five halves order in the activity. Electron diffraction and degeneracy effects are included, but the methods used currently are only valid when these effects are small. Classical methods are used to treat ion-ion interactions, so that higher order ion-ion terms are included. In the warm-matter regime heavy ions are moderately- to strongly-coupled, depending on the state of ionization, while degeneracy and quantum effects tend to keep the electrons from becoming more than moderately coupled, so that inclusion of third and possibly seven-halves order terms should be adequate.

The main improvements needed to extend ACTEX into the warm-matter region, i.e., down to $\mathrm{T} \leq \mathrm{T}_{\mathrm{f}}$ are:

- Inclusion of higher order electron-ion terms using the pseudopotential method.

- Improve the treatment of quantum statistical effects.

- Extend the theory to include plasma effects on molecules and also include, at least in an approximate way the effects of molecular clusters that are found in simulations and may be inferred by some spectroscopic data.

These improvement translate into a plan of attack: First: We will extend earlier work on pseudopotential methods to include cubic and seven-halves order electron-ion terms. We will initiate efforts to improve the quantum-statistical treatment of electrons and also to include environmental effects on molecules. Second: We will complete the quantumstatistical improvements and provide an improved version of the ACTEX code that will 
be able to predict the EOS in the dense molecular regime. New calculations of the deuterium, which can be compared to extant data, will be generated and new calculations of the aluminum Hugoniot will be performed for comparison to new data. Third: We will develop approximate methods for including clusters of atoms and molecules in the calculations. We will perform calculations of the deuterium, beryllium, and aluminum Hugoniots to validate the improvements.

\section{- Thermochemical Approach to Warm Dense Matter}

\section{(W. M. Howard and L. E. Fried)}

One of the challenges in modeling warm dense matter is finding a computationally tractable theoretical framework that can treat the transition from molecular matter to a fully ionized plasma. Ideally, such a framework should also be computationally efficient. Most current experiments in the warm dense matter regime are limited terms of spatial uniformity and timescale. These properties demand the computational modeling of WDM experiments with hydrodynamic and/or hydrodynamic-radiation transport programs. Hydrodynamic modeling requires densely spaced computational results in the form of tables, or in the form of easily evaluated numerical models.

Thermochemical codes have been used successfully in this regard to carry out the hydrodynamic modeling of high explosive detonations and gas gun-driven shocks $[1,2,3]$. These experiments generally achieve temperatures under $1 \mathrm{eV}$ and pressures under $1 \mathrm{Mbar}$. Thermochemical modeling of shock processes in this regime have been shown to be accurate to within a few percent, in terms of predicted thermodynamic properties such as pressure or temperature along the shock Hugoniot.

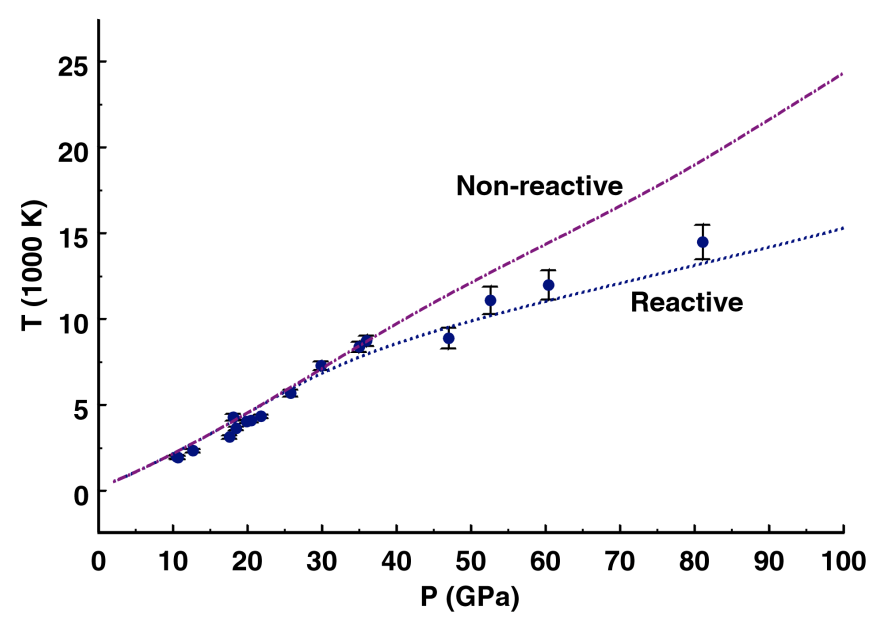

Figure: Chemical reactions in the Cheetah code accurately predict temperatures for shocked $\mathrm{N}_{2}$ up to temperatures of $15,000 \mathrm{~K}$.

A thermochemical code is a statistical mechanical model of a reactive chemical system. 
Chemical species are treated explicitly in a thermochemical code. For this reason, it is often referred to as a "chemical picture" of the reactive system. Thermochemical codes are fast, predictive, and accurate. For instance, the Cheetah code can reproduce the shock Hugoniot of dozens of materials in several seconds of computation time. This contrasts with other approaches, such as first principles molecular dynamics, where a single state point may take months of computer time on a modern parallel architecture.

The speed and accuracy of a thermochemical code, however, comes at a price. The codes depend on knowing sufficient equation of state information about the species present under the conditions of interest. This is perhaps the biggest stumbling block to applying the thermochemical approach to warm dense matter. Current experiments can give the bulk properties of matter in this regime, but not the chemical speciation. Currently, chemical speciation information may best be obtained through first principles molecular dynamics. Once a suitable database of species is built up in the thermochemical code, results may be easily extrapolated to differing thermodynamic conditions and elemental compositions from a limited number of molecular dynamics simulations.

Another challenge in the extension of the thermochemical method is the accurate treatment of ionization. The statistical mechanics of neutral molecules is handled through the construct of a spherical reference system. The actual molecules are mapped to the reference system through simple semi-empirical combination rules. This approach has proven to be accurate for a wide range of reactive systems. Partial ionization, however, is a phenomenon that is difficult to treat with traditional methods of statistical mechanics.

This difficulty may be overcome by employing a multiphase approach to the ionization transition. Namely, we can treat the free energy of the system in the WDM regime as the sum of the free energy of a neutral phase and that of an ionized phase. Minimization of the free energy will determine the relative population of the neutral and ionized state. As the temperature is increased at fixed density, the entropy will favor the ionized state and the molecular state will be depopulated. The ionized phase can be treated with any convenient non-ideal plasma theory, for example an extended Thomas-Fermi model. The molecular phase can be treated with an existing neutral species thermochemical model. There is also a possibility of adding an entropic mixing interaction between the two phases, thus avoiding possible numerical artifacts such as first order phase transitions (the so-called plasma phase transition [4]). The proposed model can be viewed as a generalization of the successful QEOS equation of state model [5]. QEOS does not track individual chemical species, and instead employs an empirical correction for bonding to Thomas-Fermi theory.

The proposed two-phase extended thermochemical model has the ability of making the transition from a neutral state of matter at room conditions to a dense plasma with pressures exceeding a Mbar and temperatures exceeding an $\mathrm{eV}$. To our knowledge, there is no current general treatment of matter that can span this regime, although some similar models have been constructed for the special case of hydrogen [4]. 
Cheetah is a thermo-chemical code that has recently been incorporated into an ALE hydrodynamics code. The Cheetah code supplies the equation of state for the hydrodynamics code. It can calculate chemical equilibrium among a variety of atoms and molecules, in a variety of phases at high pressure and temperature. Cheetah also has the capability to model chemical kinetic processes that are out of equilibrium. In using Cheetah for the equation of state, one can take advantage of fluid equation of states that have been developed for a wide variety of molecules and solids for pressure up to a few Mbars and temperatures up to $20,000 \mathrm{~K}$ The fluid equation of state is based on the exponential-6 (exp-6) potential that defines the energy of interaction of a classical fluid composed of identical spherical particles. The exp-6 potential has proven successful in modeling chemical equilibrium pressure at high pressures and temperatures. The parameters of the model have been carefully adjusted to reproduce a wide range of shock Hugoniot data up to a few Mbars, as well as low-pressure static compression and sound speed data. Cheetah also has a solid equation of state model that can account for multiphases at high temperatures and pressures.

We have used the ALE/Cheetah code to model a wide variety of single and colliding shock compression experiments. In these experiments we shock compress low-density silica aerogels, by impinging shock waves on either one or both sides of an aerogel slab. The high-pressures ( few hundred kbar) are generated by detonating high explosives. The equation of state for the aerogel in the calculation replicates shock Hugoniot data for both low-density and solid-density silica and also allows for molecular decomposition. The calculations replicate the shock waves observed radiographically in the aerogel and predict that temperatures of a few $\mathrm{eV}$ are attained in the compressed aerogel.

The current ALE/Cheetah code is a powerful tool for modeling detonation high explosives and reactive shocked materials. By adding the extensions outlined above, we can extend its validity to arbitrarily high temperatures. In fact, its accuracy will improve with temperature once the ionic phase is fully populated and the plasma becomes more ideal. The extended ALE/Cheetah code should prove useful for the modeling of NIF experiments and other experiments that probe matter under extreme conditions.

1 L. E. Fried and W. M. Howard, "An accurate equation of state for the exponential-6 fluid applied to dense supercritical nitrogen", J. Chem. Phys., 109, 7338 (1998).

2 L. E. Fried and W. M. Howard, "The equation of state of supercritical HF, HCl, and reactive supercritical mixtures containing the elements $\mathrm{H}, \mathrm{C}, \mathrm{F}$, and $\mathrm{Cl}$.", J. Chem. Phys., 110, 12023 (1999).

3 L. E. Fried and W. M. Howard "Explicit Gibbs free energy equation of state applied to the carbon phase diagram”, Phys. Rev. B, 61, 8734 (2000).

4 D. Saumon and G. Chabrier, "Fluid hydrogen at high density - the plasma phase transition", Phys. Rev. Lett., 62, 2397 (1989).

5 D. A. Young and E. M. Corey, "A new global equation of state for hot, dense matter", J. Appl. Phys., 78, 3748 (1995). 


\section{- Calculations of Near-neutral Population Kinetics}

\section{(R. W. Lee)}

In the higher temperature plasma regions of the WDM regime one is faced with the existence of neutral and near-neutral species. To theoretically access some of the most strongly coupled plasma systems one must develop the capability to predict the spectral properties of the extant species. Due to the high density and low temperature this indicates that we must employ atomic physics codes that work for neutral atoms. To determine the properties of plasma absorption and emission one first determine the equation of state or equivalently the detailed population distribution then combine this with a knowledge of the spectral characteristics of the ions and atoms as these are effected by the plasma, then piecing the various components together using a model for the plasma. Due to our focus we are interested in a spectral modeling capability that can treat plasma spectral line and edge broadening at these extreme conditions. Conventional methods for obtaining the plasma conditions from the line spectrum assume that the spectral lines are isolated from each other in wavelength space. In the WDM situations this will not necessarily be the case, as the number of elements and ion stages present may cause the lines to overlap. A method of modeling an entire section of the emission spectrum, which can then be verified by comparing to experimental spectrum to extract experimental densities and temperatures, is therefore essential.

The method of calculating a line profile correctly should entail a quantal method, or if one can verify that quantal effects are not significant then a semi-classical method can be used. However, these are both computationally expensive calculations, and require large quantities of atomic data, which may not be readily available at suitable accuracy. Thus, relatively few line profiles of few elements and ion stages have been documented in the literature. Since the range of elements and ion stages that are required will rapidly increase a method for rapid and accurate calculation of spectra is essential for both diagnostic and simulation purposes

An approach for the theoretical development of a spectral properties capability for the WDM plasmas would be to first verify that semi-classical (SC) methods can be used across a broad range of neutral and near neutral ion stages. This requires comparison of the SC method with quantal calculations. Next, we need to develop a larger scale spectral synthesis capability to model observable spectra. This requires coupling to a description of the populations.. These two parts of the efforts could develop in parallel as the synthesis model requires externally generated data. Much of that data will require the implementation of a atomic physics code, e.g., like the Superstructure suite.

With the implementation of an atomic data generating capability we could then implement a modified version of the SC model that solve as many of the detailed spectral line profile cases as possible. This is a code development and optimization effort. One would begin extending the method by developing a computational scheme based on SC and semi-empirical methods to simulate the spectrum emitted from WDM. Then implementing the SC model data in the spectral synthesis model one could perform 
comparisons with the data from WDM plasma experiments. This leads eventually to a capability that can be valid for other elements, e.g., Fe or $\mathrm{Cu}$.

\title{
- Summary of the Workshop Findings and Discussions
}

\author{
(R. W. Lee and J. D. Molitoris)
}

Creating a working understanding of the Warm Dense Matter regime requires a broadbased effort that must span the physical parameter space for matter above solid densities at temperatures near and above the Fermi energy to plasmas that are strongly coupled. The shear breadth of the parameter space indicates that several paths will have to be pursued on both the theoretical and experimental fronts.

Recent discussions and plans, including development of strategic initiatives, various workshops on WDM, the potential development of next generation sources, and possible funding by DOE/NNSA for work into the WDM regime, indicated that we, at LLNL, should come together to develop a strategy for a coherent effort in this new and challenging area. From the workshop, much of which has been outlined above, a clear path for experimental progress can be discerned. On the other hand, on the theoretical side a clear path does not yet seem to have emerged, as there was little agreement with respect to what the priorities should be amongst those interested in the calculation of material behavior.

First let us examine the situation on the experimental side. It has been discerned that there are some experiments that will not make inroads into the WDM regimes. Indeed, it was noted that impact techniques, such as gas guns and flyer-plates, do not access the appropriate temperatures. The one caveat to this statement is that extremely high-flyer plate velocities, i.e., $>24 \mathrm{~km} / \mathrm{s}$, when attained, could provide an avenue for WDM experiments. While these platforms have clearly demonstrated their utility in other regimes, they are not currently applicable to the study of WDM. Further, it was agreed that the work on Diamond Anvil Cells (DAC) and laser-heated DACs also does not reach the regimes of interest. Note that we will return later to laser-heated DAC experiments as these may provide the type of high quality data needed to validate other potentially more promising methods. Thus, we will now concentrate on other methods to reach the extreme states of matter that form the WDM regime.

For those experimental techniques that do show promise for generating the extreme states of interest in tractable experimental conditions, there are several observations. First, that for many experiments that have been proposed in WDM, there are equivalent proposals for short pulse laser based experiments. Whether the subject of interest is the measurement of opacity, EOS, spectroscopy, or any other properties in the WDM regime someone will propose that it can be done with a short pulse laser. However, there is a sparseness or lack of hard data generated from short pulse laser experiments in this 
regime. On the other hand, large-scale facilities, i.e., large-scale lasers, light sources, ion beam machines, and even high-explosive facilities, seem to provide credible avenues for performing experiments. Despite this we find that for the large-scale facilities there have, in the past, been relatively few proposals to perform work in the WDM regime.

The reason for this dichotomy may be that even though there are many short pulse lasers, there is no real user-oriented infrastructure to guide the research efforts on these facilities. As a result, efforts are often diffused by reacting to promising, high-risk areas to maintain funding. Locally, there have been several attempts to do work in the WDM regime that have dissipated. The aggressive promotion of these short pulse laser facility efforts has tended to diminish their importance. Indeed, the eventual path forward will be to merge the strengths of these accessible, "affordable" facilities with the large-scale facility capabilities.

Second, the large-scale facilities tend to be oversubscribed and strongly peer reviewed. This leads to difficulty in establishing a new field of research as the facilities are, by definition, fully subscribed. However, when experiments have made there way onto the larger facilities the results have been very positive. The prime examples of this are the $\mathrm{D}_{2}$ experiments on Nova, the synchrotron light source based effort on dynamics of heated solids up to the through the melt, and the energetic materials experiments using colliding shocks at HEAF.

The shocked $\mathrm{D}_{2}$ experiments on Nova, and now other facilities, showed that one could reach substantially higher compressions than predicted. Although this was not conceived as a WDM experiment, in fact, it turns out to be in the WDM regime. Moreover, the results have sparked, what can only be described as, religious debates amongst the various researchers trying to reproduce the results experimentally, and those attempting to calculate the response of $\mathrm{D}_{2}$ along the Hugoniot. The important point is that it took much effort and experience on laser-driven shocks to finally reach the level of sophistication to perform the experiments on the large-scale Nova systems. Meanwhile the disparate models and the lack of agreement amongst the theorists indicates that the WDM regime can represent significant challenges.

The explosively driven colliding shock experiments at HEAF were designed to address the WDM regime. It was initially thought that experiments with energetic materials were limited to temperatures less than a volt, but recent measurements show that the temperatures created are greater than $1 \mathrm{eV}$ and that the technique can be used to approach $3 \mathrm{eV}$. Conclusive density measurements are still forthcoming, but there are plans to probe the density with a high-energy heavy-ion beam and compare the result to densities extracted from radiography performed at HEAF. The point here is that these energetic material experiments were considered "high-risk", but the new results indicates that will be a high return for WDM research. Because of the size $\left(\mathrm{mm}^{3}\right)$ and time scales (microseconds) involved, the energetic material experiment are also in a position to assess equilibrium conditions, but only at the lower temperatures. However, there is overlap with the lower temperature range accessible on NIF, allowing for access to a wide range of equilibrium conditions. 
So, a pattern emerges for WDM research. The small-scale facilities are mostly laser based. These facilities are essential to define and perfect the techniques that will be imported to larger scale facilities where higher precision can be attained. Thus, we could imagine doing, laser heating on smaller systems but requiring the larger scale systems to obtain truly uniform temperature samples. Work should proceed, with small- and largescale facilities being seen as working in tandem to solve the formidable problems presented by the WDM regime. The larger facilities would include light sources, both the $3^{\text {rd }}$ generation (ALS at LBNL, APS at Argonne) and $4^{\text {th }}$ generation FEL based sources (LCSL at Stanford, TTF and TESLA at DESY), ion beam sources (particularly the well placed GSI facility at Darmstadt with its high energy petawatt laser capability), HEAF to provide detailed studies at the lower temperatures, and, of course, NIF. We believe that the pattern should be that the experimental plans for use of these facilities should be closely coupled and coordinated. Moreover, this is true for the strongly coupled plasma limit of WDM as well as the near solid density limit.

There is also a class of novel WDM experiments combining the capabilities of two experimental techniques. There are three obvious examples: First, the GSI facility in Darmstadt now has the ability to detonate up to 200 grams of high-explosive. Plans are presently underway to adapt the HEAF experiments to GSI so that the ion-beam can be used to determine density. Second, if energetic material could be used in combination with lasers, this would allow the high-explosive generated WDM to be probed with laser backlighters and utilizing laser target diagnostics. Third, the coupling of lasers to lights sources in the $3^{\text {rd }}$ and $4^{\text {th }}$ generation opens the way to both perturb and probe WDM. Finally, one could also envision other more synergistic use of experimental techniques to attain equilibrium conditions with one technique preparing the WDM and another driving it more uniformly to higher temperatures and/or densities.

On the theoretical side we have a much more confusing story. As we have outline above, there are plans for advances in the WDM regime. However, he most important issue is that we do not yet have a consensus on whether the strong shocks generated by larger lasers provide equilibrium states. It has been suggested that the strong shocks are inherently non-equilibrium, and as a result, the molecular dynamics approaches will not work. Until this is resolved it will be difficult to make progress on the simulations. The criticism from the theorists is that dense cold systems that are rapidly perturbed to create finite temperature denser systems may not have time to reach equilibrium. It is clear that making contact between a rapidly heated sample (i.e., a short pulse laser heated sample) and a decidedly equilibrium experimental method, such as a heated DAC experiment, would provide considerable understanding on the issue of equilibrium. That is, we would establish that we have a grasp on what states are attained with short pulse laser heating. Thus, because our express purpose is to forge an effort in WDM we feel it would be most useful to develop a point of contact with the finite temperature DAC measurements and a rapid heating method, e.g., laser heating of solid matter done at light sources. In this way we would heat a sample to a temperature, accessible in a DAC experiment, and perform measurements that either overlap the DAC or are complementary to them. In any case the 
measurements would need to establish the consistency, or lack thereof, between the equilibrium DAC result and the rapid heating results.

The simulations of the DAC work are considered to be valid as the DAC will come to equilibrium. Thus, the simulations can provide a crosscheck of the two methods. Further, this will also provide confidence that we understand the initial lukewarm state of matter, although not quite "extreme" and not necessarily requiring dynamic information, that makes contact with the plentiful literature on high-pressure dense matter.

Finally, the thermochemical approach to modeling WDM has made great progress in addressing the lower temperature $(<5 \mathrm{eV})$ part of the regime. One strong advantage of this approach is that it can be performed in conjunction with hydrodynamic and/or hydrodynamic-radiation transport modeling. The disadvantage, of course, is that this approach is not suitable for the higher temperature part of the WDM regime. However, work is being done to incorporate ionization into the Cheetah/ALE code and this work may help reduce confusion and develop understanding.

\title{
- Comments and Recommendations
}

\author{
(K. Budil, R. Lee, and J. D.Molitoris)
}

\section{- Comments}

It is clear that an understanding of warm dense matter is of critical importance to numerous DOE programs and the mission of LLNL, therefore LLNL should actively promote work in this regime as a programmatic activity. While a great deal of importance has been devoted to the equilibrium condition of WDM, dynamic effects have been largely unstudied to date due to the complexity of the problem. Because of this it is expected that experimental research will drive theory as we begin to bound the problem of what we do not know. Numerous current experimental facilities (pulsed power, lasers, heavy ion beams, high explosive drivers, advanced light sources) can be used to access these conditions although each facility provides different strengths and limitations. For example, current generation laser facilities can be used to access the WDM regime in small samples for short time durations, where transient effects will likely dominate the interaction. Energetic materials, on the other hand, can produce much larger samples for much longer durations, which is important to understand equilibrium and the sensitivity of materials to transition through the WDM regime. Heavy ion beams and light sources/FELs can sample larger volumes and quasi-equilibrium conditions. New facilities, like the National Ignition Facility, $4^{\text {th }}$ Generation light sources, and high flux heavy-ion accelerators have the potential to bridge the gap between transient effects and equilibrium conditions, so these facilities provide a logical advance on current efforts. Some techniques can be used both to produce WDM and diagnose it. For example, heavy-ion and laser beams could be used to determine the density and other EOS 
parameters of WDM produced by high explosive drivers. Light sources can be used to determine EOS parameters of laser produce WDM.

To move forward, tie activities together, and plan for the future, a working group should be created to provide scientific direction. This working group would be housed in the Institutes at LLNL (MRI and ILSA) and would allow us to engage the greater scientific community on this class of problems. As LLNL has a strong interest in this area of study, it is expected to be an equal partner in this initial effort, promoting a sense of community around the subject of WDM. The working group should be inclusive, with members from the labs as well as the academic community. However, for this working group to be more than a vehicle for academic discussions, LLNL must actively engage WDM as a scientific and programmatic discipline through adequate funding.

Based upon the state of current understanding of the physics in this regime, it is expected that experimental work must be initiated to motivate theoretical work. Discussions at the workshop suggested that agreeing upon a single material (perhaps $\mathrm{Be}, \mathrm{Al}$, or $\mathrm{Si}$ ) would be valuable to focus the work and allow for the assessment of different experimental techniques. For example short pulse laser techniques might be useful to highlight the effect of transient states. Energetic material experiments at the HEAF could be used to address equilibrium conditions in WDM. Such experiments can help bound the question of what is not known and serve as a starting point for theoretical investigations.

For LLNL to assume a lead role in WDM research, we need to utilize the present capabilities of off-site experimental techniques (such as the present generation light sources and heavy-ion facilities) in WDM research and prepare for the use of the next generation facilities. These include NIF, $4^{\text {th }}$ Generation Light Sources / FELs, and the upgraded Heavy-ion capabilities of GSI.

\section{- A Path for LLNL/DOE/DP}

The idea is to get LLNL involved in the development of experiments preparing for the next generation facilities as soon as possible so that we can be well positioned to obtain data of importance to the program when they are up and running. The measurement of EOS and opacity in the WDM regime will best be performed at the next generation of light sources and the upgraded GSI heavy-ion facility. By involving ourselves in present experimental work we can lay the foundation for scientifically sound future involvement. On one hand, if we are not involved in the development of these facilities we will not be in a position to participate fully. On the other hand, we have a role to play through the development and implementation of techniques (lasers, diagnostics, WDM explosive drivers, etc.) that can augment these new facilities and provide a position that will allow us to share fully in the use of them. LLNL, DOE, and DP/NNSA are already involved to a certain extent. The new Phelix petawatt laser at the GSI is relying heavily on equipment from the Nova laser and the DOE Office of Science is involved at several levels. The future combination of the Phelix laser, high-intensity heavy-ion beams, and a small explosive firing tank at one facility allows many options for the generation and 
diagnosis of WDM. In fact, LLNL scientists are already involved with the laser and high-explosive aspects of this facility.

We should encourage collaborations with these facilities that serves the programmatic goals. As the interaction with these external facilities tends to be judged on a scientific basis we can assist by providing the facilities with a new and exciting set of scientific motivations for their unique capabilities. The correct combination is the new, and challenging regime of WDM, or more globally finite temperature dense matter, coupled to LLNL scientific strengths. This would, for example, mean our laser and laser diagnostic capabilities coupled to FELs. This also means our expertise in the development of explosive WDM experiments coupled to heavy-ion diagnostic probes. This means taking a lead role in foreign collaborations for these experiments. This is at the core of the programs that DOE/DP and LLNL are pursuing. The idea is quite simple and the path straightforward.

For the SSMP to benefit the most, LLNL needs to be the leader in WDM research, which means we need to move on all three future fronts: NIF, light sources, and heavy-ions. LLNL is the site for NIF, and this will come most naturally as the funding is there. For the other two fronts, we need to develop funded efforts. This will require significant programmatic effort, most likely beyond the scope of LDRD.

The situation with GSI is clear. We have been offered beam time in the coming year through their Plasma Physics Group and have the opportunity to lead a collaboration with GSI, TU Darmstadt, and the Russians in WDM. They have recognized that the WDM source developed at HEAF is a viable source to study WDM with ion beam energy loss techniques and with the Phelix laser. For a small investment LLNL can further develop WDM with energetic materials at HEAF and lead this collaboration. This opportunity exists now.

For light sources, we team, at first, with our current allies and collaborators. These include LBNL/ALS and UCB/Physics to develop the short pulse laser based capabilities that are coupled to light sources. The current state-of-the-art is being pursued at the ALS beamline 5.3.1 in concert with LLNL. The path towards an advance sub picosecond light source capability has three avenues. First, there is the proposed slicing source at the ALS that will provide a short pulse capability on the existing ALS. This is of great interest as it provides an experimental advance on a setup that is currently in operation. Second, the interim source SPPS at SLAC will become available soon. This source will be the first sub-picosecond (80 fs) light source to come online. It proximity to LLNL and the potential to perform the first experiments in the WDM regime would allow us to perform the necessary preliminary research on dual sub-picosecond beam synchronization, subpicosecond x-ray diagnostic development, and experimental development of WDM EOS and opacity experiments. Third, the second phase of the TTF facility at DESY, an FEL, will become operational in 2004. Meetings have been held, working groups have formed at workshops, and Proposals have been written to initiate a beamline dedicate to finitetemperature dense matter studies. All of these avenues provide the real possibly of 
timely progress in the WDM regime pursuant to the eventual operation of full $\mathrm{x}$-ray FELs at SLAC and DESY.

All of these activities fold into the LLNL WDM working group, which will keep us tied to the program and to future work on NIF. The plan is clear, the chances of success are extremely high, it is the will to start that we now require. There is no better time than now. 